\title{
Crescimento Econômico e as Políticas de Distribuição de Renda e Investimento em Educação nos Estados Brasileiros: Teoria e Análise Econométrica ${ }^{\star}$
}

\author{
- Jollson Dias* - Maria Helena Ambrósio Dias*
}

\begin{abstract}
RESUMO
O objetivo deste artigo é desenvolver e testar empiricamente um modelo de crescimento econômico que incorpore educação, distribuição de renda e nível tecnológico. Nos testes empíricos, uma das variáveis importantes é a razão das escolaridades dos empregados e empregadores. Além desta é utilizada uma medida de tecnologia e os seguintes índices de desigualdade de renda: i) participação na renda dos $40 \%$ mais pobres; ii) participação na renda dos $10 \%$ mais ricos; iii) participação na renda da classe média; e iv) o coeficiente de Gini e duas outras variáveis de controle: v) a percentagem de trabalhadores autônomos; e vi) a taxa de desemprego. A econometria de painel de dados empregada consiste de testes de raiz unitária, para verificar a estacionariedade dos dados, heterogeneidade, autocorrelação, dependência seccional, e as estimativas de efeitos fixos, randômicos e dinâmicas. Os resutados demonstram que a taxa de crescimento da produtividade dos Estados brasileiros é afetada positivamente: a) pelo aumento na razão do nível educacional dos empregados vis-à-vis empregadores; b) por políticas de redistribuição de renda que favoreçam os $40 \%$ mais pobres e a classe média; e c) pela tecnologia importada. Contudo, os investimentos em educação dos Estados brasileiros possuem efeitos negativos sobre a taxa de crescimento de suas produtividades, estes agem como um imposto realocativo dos insumos do setor produtivo de bens para o de educação no curto prazo.
\end{abstract}

\section{PalaVRas-CHAVE}

crescimento econômico, distribuição de renda, educação e análise em painel de dados

\begin{abstract}
The objective of the paper is to develop and empirically test an economic growth model that incorporates education, income distribution effects and technology. In the empirical tests, one of the driving forces of productivity growth is the ratio of the average knowledge levels of employees and entrepreneurs. Besides this variable and a measure of technology the following variables are considered: $i)$ the income index of the $10 \%$ richest; ii) the income index of the $40 \%$ poorest; iii) middle class income size index; iv) the Gini coefficient and two control variables: v) self employed and vi) the unemployment rate. The econometrics of panel data employed includes panel tests for stationary, heterogeneity, auto-correlation, cross-section dependency, and fixed, random and dynamic effect estimates. The overall result shows that the productivity growth is influenced positively by: a) the increase in the employee-employers education level; $b$ ) income redistribution policies that benefits middle classes and the $40 \%$ poorest in the economy; c) the imported technology. Nevertheless, the investment in education made by the Brazilian States has a negative impact on their productivity growth rate. It may behave as shift tax that allocates inputs from production of goods to the human capital accumulation sector.
\end{abstract}

\section{KEY WORDS}

economic growth, income distribution, education and panel data analysis

\section{JEL Classification \\ O15, C33}

- Os autores agradecem imensamente o apoio financeiro recebido do CNPq -Conselho Nacional de Desenvolvimento Científico e Tecnológico, além dos comentários dos pareceristas anônimos.

* Professores Titulares do Departamento de Economia da Universidade Estadual de Maringá. E-mails: jdias@uem. br; mhadias@uem.br. End. para contato: Universidade Estadual de Maringá - Departamento de Economia - 87020900 - Maringá - PR.

(Recebido em setembro de 2005. Aceito para publicação em março de 2007). 


\section{INTRODUÇÃO}

As políticas econômicas, como as de melhoria do capital humano e da distribuição de renda, objetivam o crescimento da produtividade de longo prazo e são avaliadas em nível de países. Neste aspecto, a literatura apresentada aqui destaca o papel positivo exercido pelo capital humano e o papel indefinido exercido pela distribuição de renda nos países. Em nossa visão, os fatores que dificultam a análise em nível de países são as diferenças entre suas estruturas físicas e organizacionais, sociais e culturais e, principalmente, suas políticas econômicas. Este conjunto de diferenças implica resultados explicativos para os efeitos da desigualdade sobre o crescimento econômico ambíguos, ou de difícil interpretação. Neste artigo, ao enfocar os Estados brasileiros, os efeitos das diferenças nos resultados são minimizados, permitindo que aspectos quantitativos de suas políticas econômicas específicas sobre o crescimento da produtividade sejam analisados.

Apesar de focalizar aspectos econômicos dos Estados, os efeitos remanescentes, como características específicas de infra-estrutura, população, organização de mercado de trabalho etc., são captados por meio da proposição de variáveis indiretas e de técnicas econométricas especializadas.

Neste contexto, o artigo se propóe a desenvolver e testar empiricamente um modelo teórico que considera quatro principais aspectos geradores do crescimento da produtividade no médio e longo prazos: i) investimento em educação; ii) distribuição de renda; iii) níveis de capital físico e de tecnologia; e iv) tecnologia importada.

A escolha dessas variáveis considera que os investimentos em educação, no médio e longo prazos, resultam em aumentos de capital humano e, portanto, geram aumentos de produtividade. Por outro lado, a participação do setor público nesse processo, por meio da geração de impostos, pode criar custos econômicos e sociais em sua fase inicial e, então, funcionar como inibidor do crescimento econômico. A avaliação da existência ou não destes custos iniciais pode identificar por que há investimentos diferenciados sendo realizados em educação entre os Estados. Além disso, o acesso a investimentos em educação por diferentes classes sociais provavelmente ocorre de forma não equitativa, especialmente entre as classes de trabalhadores e empresários. Desta forma, o capital humano acumulado entre estas duas classes pode ter impactos diferenciados no aumento de suas produtividades, inclusive com reflexos na produtividade agregada dos Estados e na evolução da distribuição de renda, havendo assim a necessidade de políticas mais específicas para os investimentos em educação, que consideram a forma diferenciada de acesso entre as classes. 
Outro aspecto importante da motivação deste artigo está na avaliação, a posteriori, dos ganhos de produtividade, que são refletidos nos índices de distribuição de renda. Esses índices são uma imagem de como a sociedade distribui a posteriori seus ganhos realizados e, portanto, podem agir como fator contribuidor ou não de novos aumentos no futuro. Por exemplo, se os ganhos de produtividade estiverem concentrados de alguma forma em uma classe específica da sociedade, as demais classes podem se sentir desobrigadas a contribuir para aumentar a produtividade como um todo. Dessa forma, se faz premente avaliar se os efeitos de políticas de redistribuição de renda são ou não benéficos no médio e longo prazos, e que classe deve ser beneficiada para atingir um efeito maior no crescimento da produtividade.

Além disso, a tecnologia se coloca como elemento relevante para a produtividade. Os investimentos em tecnologia realizados pelos Estados diferem e, portanto, exercem efeitos diferenciados sobre os estoques existentes de capital humano, físico, infraestrutura em geral etc. Apesar de se reconhecer teoricamente o papel da tecnologia e de seus impactos diferenciados na produtividade dos Estados, a não-existência de medidas diretas para esta variável tende a dificultar as análises econômicas. Assim, na falta de uma medida direta, utiliza-se uma medida indireta dos investimentos em tecnologia, de tal forma que o problema de omissão de variável seja amenizado. As medidas indiretas utilizadas são de nível e de fluxo. A primeira implica a construção de um indicador agregado do estoque de capital físico e tecnologia. Esta variável tem como principal função estabelecer as diferenças estruturais e potencialmente organizacionais das economias dos Estados. A segunda medida é representada pelo fluxo de importações de tecnologia de cada Estado como proporção de seus respectivos PIB (Produto Interno Bruto). Esta última tem a função de verificar o papel da interação das economias estaduais com as tecnologias desenvolvidas no exterior.

Dessa forma, este artigo procura contribuir com a literatura, abordando esses aspectos diferenciados do investimento em educação, da distribuição de renda e do investimento em tecnologia, em nível teórico e empírico, para os Estados brasileiros. A breve discussão da literatura, na próxima seção, verifica que os efeitos da desigualdade de renda no crescimento econômico geram resultados contraditórios, devido à não observância de algumas condições técnicas, como estacionariedade das variáveis, variáveis omitidas e efeitos fixos, condições estas que são minimizadas neste trabalho. Outro aspecto que merece atenção refere-se aos testes dos efeitos do capital humano (escolaridade) no crescimento econômico. Apesar dos diversos conceitos empregados na literatura, nenhum considera o capital humano dos empregados e dos empregadores de forma distinta, como proposto neste artigo. Outro aspecto, considerado neste artigo, se relaciona ao problema da omissão da variável nível tecnológico que, apesar de discutida na literatura, não havia sido formalmente considerada. Assim, este artigo propõe uma forma de se considerar esta variável em estimativas de crescimento econômico. 
As demais seções do artigo abordam os seguintes aspectos: a seção 2 situa este trabalho na literatura nacional e internacional; a seção 3 apresenta um modelo teórico classificando dois tipos de capital humano, o dos empregados e dos empregadores, considerando a distribuição de renda, os níveis de tecnologia e o acesso à educação entre as classes; a seção 4 aborda a análise dos painéis de dados, na qual testes de estacionariedade, heterocedasticidade, autocorrelação e de especificação permitem a escolha do melhor modelo e da técnica de estimativas econométricas; e a última seção contém a conclusão.

\section{BREVE REVISÃO DA LITERATURA}

Especificamente com relação à literatura brasileira, a seleção de estudos sobre a desigualdade de renda e crescimento econômico do Brasil procura destacar aqueles que complementam este trabalho por oferecer resultados dos efeitos de políticas econômicas sobre as variáveis de interesse. Desta forma, esses estudos servem de balizadores para políticas econômicas específicas para os Estados brasileiros, com o intuito de reduzir a desigualdade de renda e, conseqüientemente, aumentar o crescimento econômico.

Os trabalhos relacionados à desigualdade de renda no Brasil têm marco inicial com Langoni (1973). Este autor observou que a desigualdade da renda agregada estava relacionada a uma medida de decomposição dos salários em setores da economia. Este trabalho foi importante, pois gerou dois grupos de estudos. O primeiro grupo concentrou-se no estudo da evolução da distribuição da renda, utilizando diferentes técnicas, como por exemplo: Bacha e Taylor (1978), Hoffman (1989), Bonelli e Sedlacek (1989), Barros e Mendonça (1992), Ramos (1993), Calmon et alli. (2000) e Elbers et al. (2004). O segundo grupo, por sua vez, procurou por explicações quanto ao comportamento da desigualdade de renda no tempo. Dentre estes trabalhos, destacamos os seguintes: Ramos (1995), em que a desigualdade de renda é explicada em parte pela desigualdade dos salários das indústrias; Calmon et al. (1999), em trabalho posterior, associaram as desigualdades de renda, em especial as desigualdades salariais nas indústrias, ao crescimento do PIB brasileiro, observando que as oscilações econômicas eram os fatores explicativos destas desigualdades de renda; Barros e Mendonça (1996), no qual o mercado de trabalho e suas inter-relaçóes exercem papel preponderante na distribuição de renda; Leal e Werlang (1991), Ferreira (1999) e Ferreira e Litchfield (2001), que apresentaram a forte associação existente entre educação e desigualdade de renda no Brasil; Cardoso, Barros e Urani (1995), que destacaram os papéis que o desemprego e a inflação exercem na desigualdade de renda; Azzoni e Servo (2002) combinaram as teorias de capital humano, segmentação e desenvolvimento polariza- 
do para explicar a desigualdade de renda. Apesar da importância marginal igual das variáveis educação, diferenças regionais e características pessoais, a desigualdade remanescente é em grande parte explicada pelas diferenças regionais presentes na rigidez dos mercados de trabalhos; e, finalmente, Hoffman (2005) em seu estudo sobre o papel das políticas de transferências de renda concluiu que estas não foram as principais causas da queda da desigualdade de renda no Brasil, colocando como explicação possível que estas ainda não foram suficientes para atingir o objetivo proposto.

Os trabalhos recentes que avaliam mais extensivamente a relação existente entre desigualdade de renda, crescimento econômico e políticas econômicas estão embasados em simulações quantitativas. Por exemplo, Bourguignon (2002) realizou microssimulações com microdados para o Brasil e as comparou com outros países. Segundo ele, a desigualdade de renda no Brasil esteve associada à questão do baixo crescimento no período 1976-1996, em que se gerou menos emprego e mais informalidade no mercado de trabalho. Kakwani, Neri e Son (2006), usando simulações de políticas de crescimento econômico pró-pobres, concluíram que políticas de benefícios sociais direcionadas aos pobres geram aumentos no crescimento econômico do Brasil. Ainda no contexto de simulações, para Almeida e Guilhoto (2006), a melhoria no crescimento e na desigualdade de renda pode ser conseguida com políticas específicas que beneficiem o crescimento de dois setores da economia brasileira: indústria têxtil e siderurgia.

$\mathrm{Na}$ avaliação específica do crescimento econômico dos Estados brasileiros, o principal objetivo de nosso estudo, há dois trabalhos que se destacam: o de Zini Jr. (1998) e Mossi et alli (2003). O primeiro, utilizando indicadores de capital humano, níveis de capital e tecnologia e indicadores socioeconômicos, verificou que o crescimento econômico dos Estados brasileiros está atrelado aos investimentos em capital humano, na indústria em geral e na infra-estrutura, com efeito negativo para a concentração da propriedade agrícola. A medida indireta de integração tecnológica utilizada pelo autor foi de exportações dos Estados, que não apresentou significância estatística. Portanto, políticas econômicas que enfoquem especificamente estas áreas beneficiariam o crescimento econômico das unidades da federação e, por conseguinte, do Brasil como um todo. O segundo trabalho, técnicas de estudos espaciais, avaliou a questão da geografia econômica dos Estados, em especial o efeito transbordamento de agrupamentos regionais. Os resultados sugerem que o crescimento econômico dos Estados é parcialmente determinado por condições espaciais, em que Estados próximos a Estados desenvolvidos tendem a ser mais prósperos devido ao efeito de transbordamento.

É interessante destacar dois aspectos no trabalho de Zini Jr. (1998). Primeiro, o autor utilizou como indicadores do capital humano dos Estados as percentagens de pessoas analfabetas e com escolaridade acima de oito anos. Segundo, o autor utilizou nas 
estimativas da sua regressão estrutural a técnica de pooled dados (junção de dados de corte e séries temporais) e considerou os efeitos das diferenças regionais através de variáveis indicadoras, dummies. Neste sentido, o nosso trabalho é uma continuidade em relação ao trabalho de Zini Jr. (1998) ao avançar nos seguintes aspectos: i) construção de um modelo teórico; ii) variáveis de medidas de capital humano e tecnologia; iii) considera o papel da distribuição de renda; iv) utiliza novas técnicas econométricas em painéis de dados, que consideram os efeitos fixos dos Estados; e, v) no tratamento da estacionariedade das séries.

Retornando ao escopo dos trabalhos acima, podemos afirmar que, em síntese, estes destacam a necessidade de políticas mais efetivas para combater a desigualdade de renda e aumentar o crescimento econômico. Estas políticas incluem investimentos em educação, transferências de renda, benefícios sociais, investimento em infra-estrutura, políticas que amenizem ciclos econômicos e/ou que beneficiem setores específicos da economia, como a formação de clusters (agrupamentos). Mas, para que estas políticas sejam válidas, se faz necessário encontrar uma associação eficaz entre distribuição de renda e crescimento econômico para os Estados brasileiros, e este é um dos principais objetivos deste trabalho. Para dar início à busca desta associação, efetuamos uma breve revisão da literatura internacional que expomos a seguir.

A literatura internacional que trata da relação entre desigualdade de renda e crescimento econômico iniciou com o artigo de Kuznets (1955). A relação na forma de $\mathrm{U}$ invertido, encontrada pelo autor, estabeleceu como primeira fase a ocorrência de maiores taxas de crescimento econômico associadas a aumentos na desigualdade de renda. A segunda fase tem como característica principal que maiores taxas de crescimento estão associadas à redução na desigualdade de renda. ${ }^{1}$

A inversão desta relação tornou-se objeto de estudo mais intenso quando Alesina e Rodrick (1994) avaliaram as conseqüências da desigualdade de renda para o crescimento econômico. Segundo os autores, a desigualdade de renda produz uma redução na taxa de crescimento da economia de longo prazo. Portanto, as intervenções através de políticas de redistribuição de renda são benéficas para o crescimento da economia. As variáveis utilizadas nos testes econométricos incluíram uma proxy para capital humano, número de alunos matriculados no primário e o índice de Gini. A mesma análise de causalidade entre a desigualdade de renda e a taxa de crescimento da economia foi efetuada por Person e Tabellini (1994). O principal aspecto inovador do artigo foi a variável capital humano. A construção desta variável foi melhorada através da soma da percentagem de matriculados nos níveis de educação primário, secundário

l É interessante destacar o estudo para o Brasil de Azzoni (2001). Em sua avaliação da convergência de renda dos Estados brasileiros, estimou a curva de Kuznets. Quando considerou a renda relativa dos Estados, verificou que os Estados pobres estão sempre na parte ascendente da curva e os ricos, na parte descendente. 
e terciário. O impacto positivo desta proxy de capital humano - que também é uma medida do investimento educacional realizado no sistema - sobre a taxa de crescimento econômico demonstrou a importância deste investimento para o crescimento das economias. Outro detalhe importante foi a substituição dos tradicionais índices de desigualdalde de renda, como o de Gini, por uma espécie de diferença entre os vários produtos agregados dos países (PIB) em relação ao do país com o maior PIB. Este diferencial demonstrou ser negativamente relacionado com a taxa de crescimento da economia. Portanto, o resultado foi que, quanto maior a economia, maior é sua taxa de crescimento.

Ainda no estudo dos efeitos da distribuição de renda, temos outra inovação na utilização de variável relacionada à medida de distribuição de renda. Easterly (2001a) utilizou o tamanho da classe média, percentagem da renda detida pelos indivíduos entre o quarto e o sexto decil, para verificar os efeitos sobre o crescimento econômico de longo prazo. Segundo o autor, a associação positiva encontrada entre ambos demonstra a importância de políticas que favoreçam a distribuição de renda em favor da classe média.

Outro aspecto da má distribuição de renda nas economias está relacionado ao nível de impostos. Segundo Alesina e Perotti (1994), níveis elevados de desigualdade de renda geram políticas de transferência crescente de renda para minimizar estes mesmos indicadores. Uma conseqüência destas políticas é o aumento nos impostos que, por sua vez, tem impacto negativo na taxa de crescimento da economia. ${ }^{2}$ Esta hipótese foi testada empiricamente por Milanovic (2000) para uma amostra de 79 países, tendo confirmado que maior desigualdade de renda causa aumentos nas políticas de redistribuição de renda via impostos e, portanto, menores taxas de crescimento econômico.

O modelo teórico aqui desenvolvido procura incorporar o efeito dessa associação entre maior desigualdade de renda e maior taxação na economia. Portanto, o desenvolvimento de uma teoria que associe ambos os elementos pode auxiliar a compreender os efeitos perniciosos da distribuição de renda dos Estados brasileiros.

Por mais coerentes que possam parecer os efeitos negativos da desigualdade de renda sobre a taxa de crescimento, não existe no entanto um consenso sobre estes efeitos. No que se refere aos dados sobre o assunto, Deininger e Squire $(1996,1998)$ realizaram esforços para a construção de uma base de dados de desigualdade de renda para os países. Em seus resultados, os autores não encontraram uma relação de impacto significante entre desigualdade de renda e crescimento econômico.

2 Alesina e Perotti (1994) oferecem uma explicação adicional. Segundo os autores, um alto nível de desigualdade de renda também reduz o crescimento futuro porque gera instabilidade política, aumentando as incertezas, que prejudicam os investimentos na economia. 
Dentro desse contexto e em nível de países, controlando os efeitos fixos para os mesmos, há o trabalho realizado por Banerjee e Duflo (2003). Os autores utilizaram os dados de Barro (1998) e Perotti (1996). De acordo com Banerjee e Duflo, mudanças no índice de Gini para qualquer direção reduzem crescimento econômico. Este resultado foi obtido considerando diferenças nas políticas dos países e diferenças nas distribuições dos parâmetros.

Mais recentemente, Forbes (2000), também controlando para os efeitos fixos dos países, estimou a relação acima descrita sob várias especificações econométricas. Os efeitos do índice de Gini de desigualdade de renda dos países sobre a taxa de crescimento da renda per capita da economia foram positivos e altamente significantes. A inovação do autor foi separar a definição do capital humano entre nível de escolaridade masculina e feminina. Nesta especificação, o nível de escolaridade masculina apresentou efeito positivo para a taxa de crescimento econômica nos modelos, nos quais teve coeficiente estatisticamente significante. Porém, o nível de escolaridade feminina apresentou influência negativa na maioria das especificações.

Apesar de aplicar os efeitos fixos no método de painel de dados, estes trabalhos não consideraram alguns aspectos econométricos, tais como o problema de estacionariedade das variáveis e o de autocorrelação. No primeiro caso, pode ter ocorrido o problema de regressão espúria e, no segundo, coeficientes viesados. Neste último caso de autocorrelação, o estudo de Forbes (2000) admite explicitamente que seus resultados podem ter recebido influência do problema.

Especificamente para um país, com destaque para estudos das unidades da federação dos Estados Unidos, Partridge (1997) demonstrou que a taxa de crescimento da renda per capita para os Estados americanos possuía uma relação positiva com o índice de distribuição de renda de Gini e com o percentual de matriculados no ensino médio (high school) e superior (college). As variáveis de controle usadas foram: o tamanho dos setores de construção, indústria, transporte e finanças; o tamanho do governo local; a percentagem da participação das políticas de melhoria de bem-estar; e o desemprego. Assim, uma vez controlados os fatores fixos dos Estados, a relação entre crescimento e desigualdade seria positiva. De acordo com Partridge (1997) e Panizza (2002), utilizando métodos econométricos que consideram efeitos fixos, não foi obtida nenhuma relação significante entre desigualdade e crescimento econômico para os mesmos Estados americanos. Este resultado também foi confirmado recentemente por Partridge (2005). Mais especificamente neste último, os resultados indicaram uma relação conflitante entre desigualdade e crescimento para os Estados americanos, quando foram aplicadas técnicas econométricas que controlaram os efeitos fixos dos Estados. 
Esse resultado conflitante foi corroborado pelo estudo efetuado por Castello e Domenech (2002), aplicado em nível de países. Quando consideraram somente medidas de desigualdade de capital humano, encontraram uma relação inversa com o crescimento econômico. No entanto, quando os autores consideraram medidas de desigualdade de renda e de capital humano em conjunto, desigualdade de renda e crescimento econômico apresentaram relação estatística positiva.

Por outro lado, entendemos que a explicação para esta relação conflitante pode estar associada com a relação existente entre acumulação de capital humano e desigualdade. Assim, os efeitos benéficos da acumulação de conhecimento de longo prazo podem estar sendo minimizados por uma política de taxação de renda para investimentos em educação pública. Dessa forma, uma combinação de acesso à educação diferenciada entre classes gera desigualdades, que, por sua vez, geram aumento nos tributos com a finalidade de reduzir estas desigualdades, as quais acabam por reduzir a taxa de crescimento. Em consonância com estes resultados, o estudo efetuado por Walde (2000) os confirma, enfatizando que maior acesso por parte da elite da sociedade no sistema educacional corrobora um aumento na desigualdade de renda. Isto porque existe uma relação entre desigualdade de educação e de renda, conforme Lam e Levinson (1991), e, portanto, requer que os investimentos em educação sejam eficientes e equânimes para que possam reduzir a desigualdade de renda existente. (Souza, 1994). Ou seja, espera-se que maior investimento público em educação possa reduzir a desigualdade de renda gerada em função do próprio acesso à educação de forma diferenciada entre classes sociais. (Sylvester, 2002). Neste aspecto, o modelo aplicado aqui capta este acesso diferenciado dos empregados e empregadores, verificando após este controle as implicações da relação entre crescimento econômico, distribuição de renda e os investimentos em educação.

Com relação ao investimento público em educação, os seus efeitos sobre crescimento econômico foram inicialmente estudados por Easterly e Rebelo (1993) e, mais recentemente, por Sylvester (2000). As respostas encontradas pelos autores são que os investimentos em educação não estão relacionados à taxa de crescimento em algumas especificações, e em outras tal relação foi negativa. Similar visão foi obtida por Sylvester (2002), o qual verificou que desigualdade de renda gera aumento de investimentos em educação, apresentando efeito negativo sobre a taxa de crescimento da produtividade no curto prazo; porém, no longo prazo, tem efeito positivo na taxa de crescimento por este causar aumento nos níveis educacionais. No entanto, Wolf (2000) verificou que os níveis educacionais e o crescimento do número de matriculados, uma proxy para o investimento em educação, com a exceção do ensino básico de quatro anos (elementary school), não são significantes para influenciar a taxa de crescimento da produtividade dos trabalhadores. 
Em resumo, as implicações da distribuição de renda e do investimento em educação para o crescimento econômico ainda necessitam de comprovação econométrica, segundo a literatura internacional. Isto porque esta nos informa que políticas econômicas sugeridas por estudiosos do Brasil - de transferência de renda e investimentos em educação - possuem custos para amenizar a desigualdade de renda e gerar crescimento econômico. Esses custos são, em especial, de aumento da carga tributária, que pode implicar menor crescimento econômico no curto prazo. No entanto, temos que estabelecer se a relação entre crescimento econômico, distribuição de renda e as políticas que os afetam, como de investimentos em educação, são benéficas para o caso dos Estados brasileiros. Portanto, esperamos que, com o modelo teórico e a metodologia econométrica empregadas neste estudo, estabeleça-se tal relação para os Estados brasileiros de forma aceitável, e que, assim, contribua com a literatura no assunto.

Neste contexto, nosso estudo, além da inovação apresentada para a medida de capital humano (se comparado aos estudos mencionados) e dos recursos permitidos pela aplicação econométrica, utiliza outras medidas de desigualdade de renda para os Estados brasileiros e o tradicional índice de Gini. A razão é que o índice de Gini é uma medida agregada mais ampla e, portanto, não enfatiza alterações nos limites superiores e inferiores da distribuição de renda. Assim, as medidas a serem consideradas são as percentagens de rendas detidas pelos $40 \%$ mais pobres, pela classe média e pelos $10 \%$ mais ricos.

Segundo Temple (1999), outro problema comum em estudos de crescimento econômico deste gênero é o de variáveis omitidas, especialmente a variável nível tecnológico. Neste aspecto, nosso estudo procura oferecer uma solução para os problemas das estimativas em painéis de dados para os Estados brasileiros, ao considerar duas variáveis para representar nível tecnológico dos Estados brasileiros.

A seguir apresentamos o nosso modelo teórico, que nos permite compreender melhor as relações a serem estudadas.

\section{O MODELO TEÓRICO: CRESCIMENTO, DISTRIBUIÇÃO DE RENDA, EDUCAÇÃO E NÍVEL TECNOLÓGICO}

O modelo a ser desenvolvido aqui segue o de Person e Tabellini (1994) com relação à incorporação da distribuição de renda como um importante elemento na determinação de uma política de taxação nas economias. No entanto, a definição de distribuição de renda aqui presente foi ligeiramente modificada e compreende somente duas classes: empregados e empregadores. Com esta modificação, esperamos obter uma compre- 
ensão maior dos efeitos da distribuição de renda e sua influência sobre o crescimento econômico.

Outro aspecto importante do modelo é considerar dois tipos de capital humano, conforme proposto por Dias (2001). Ressalvamos que o modelo teórico não explicita formalmente a acumulação de capital físico, ou seja, admite-se ser esta exógena e, portanto, concentra-se nos aspectos de interesse, que é a relação entre o crescimento da produtividade, a desigualdade de renda e os níveis educacionais dos empregados e empregadores.

A função de produção agregada postulada para a economia possui a seguinte forma:

$$
y=A h^{\alpha} k^{1-\alpha}
$$

Na qual $y$ é a produção por trabalhador; $A$ representa o nível tecnológico e de capital físico; $h$ é o nível do capital humano do trabalhador; $k$ é o nível do capital humano dos empregadores; e $\alpha$ é o parâmetro da função de produção. A economia produz um único produto que é utilizado para consumir e investir em diferentes tipos de capital humano.

Além disso, a infra-estrutura educacional recebe investimento do governo, que tem suas receitas através da tributação da produção agregada da economia (equação (1)). O nível de taxação da economia depende da distribuição de renda prevalecente entre as classes: empregadores e empregados. Portanto, o agente econômico com maior nível de ativos paga mais impostos. Assim, a política de taxação para estes agentes econômicos é dada pela seguinte fórmula:

$$
g=\tau\left(\frac{a_{i}}{a_{m}}\right) \text { ou } g=\tau \Omega_{i} ; \text { sendo } \Omega_{i}=\frac{a_{i}}{a_{m}} \text { e } a_{i}=w, k
$$

Na equação (2), w representa o trabalhador e $k$ o empregador. Se o agente econômico detentor da renda $\left(a_{i}\right)$ detivesse renda maior do que a média das rendas $\left(a_{m}\right)$, então este pagaria mais impostos e, portanto, em termos agregados, o nível de taxação seria maior. Se a razão for idêntica, então os impostos seriam eqüitativos sobre os rendimentos dos empregadores e empregados. Esta especificação captura o seguinte mecanismo: em sociedades onde impera desigualdade de renda, o nível de taxação em termos agregados será maior.

Dessa forma, os fatores que alteram o valor de $A$ no tempo são: a tecnologia, desenvolvida externamente $(\phi)$, o que significa que esta não depende de investimentos da própria economia para seu desenvolvimento; e os investimentos em educação $(\delta)$. Para 
simplificar, vamos nos concentrar somente na parte dos impostos que são arrecadados e aplicados em educação. Portanto, assumindo que o custo de arrecadação e intermediação de impostos inexiste e que estes impostos são totalmente investidos em educação, então $\mathrm{A}=\mathrm{f}(\phi, \delta) .{ }^{3}$ Substituindo esta última definição e a equação (2) na equação (1), a renda disponível per capita da economia apresenta a seguinte representação:

$$
y=\left(1-\tau \Omega_{i}\right) f(\varphi, \delta) h^{\alpha} k^{l-\alpha}
$$

Assumindo que esta economia é dominada por empresas perfeitamente competitivas, a maximização de lucros implica:

$$
\begin{aligned}
& w h=\alpha\left(1-\tau \Omega_{i}\right) f(\varphi, \delta) h^{\alpha} k^{1-\alpha} \\
& \pi=y-w h=(1-\alpha)\left(1-\tau \Omega_{i}\right) f(\varphi, \delta) h^{\alpha} k^{1-\alpha}
\end{aligned}
$$

O rendimento do trabalhador representativo $(w h)$ (equação (4)), e a renda do empregador representativo $(\pi)$ (equação $(5)$ ), dependem dos níveis do capital humano, da tecnologia exógena, do investimento em educação e da distribuição de renda. Esta última tem um efeito negativo sobre as equações de acumulação ao produzir uma maior taxação das rendas.

A dinâmica desta economia é determinada pelos dois agentes econômicos representativos, em que cada agente acumula independentemente seu respectivo capital humano. Apesar da função de bem-estar social de ambos possuir parâmetros idênticos, cada uma pode diferir com relação à quantidade a ser consumida, bem como na determinação do capital humano a ser acumulado. Esta simplificação do processo dinâmico permite obter os resultados desejados sem incorrer em perda de generalidades. $\mathrm{Ou}$ seja, a admissão de parâmetros diferenciados na função de bem-estar não agrega informações relevantes para o objetivo do trabalho. Assim, a função de bem-estar $(W)$ é a seguinte:

$$
\begin{aligned}
& W=\int_{0}^{\infty} \frac{\left(\mathrm{c}_{\mathrm{j}}\right)^{1-\xi}-1}{1-\xi} e^{-\rho t} d t \quad \text { para } \xi \neq 1, \mathrm{e} \\
& W=\int_{0}^{\infty} \ln \left(\mathrm{c}_{\mathrm{j}}\right) e^{-\rho t} d t \quad \text { para } \xi=1 .
\end{aligned}
$$

3 Esta hipótese não é crucial para o modelo, uma vez que a forma da função assumida é genérica e, portanto, não precisa ser necessariamente monotômica em relação à arrecadação de impostos e seu destino. 
Na função de bem-estar acima, $c_{j}$ é o consumo, sendo que $j=w, k$ representa o consumo dos trabalhadores $(w)$ e dos empregadores $(k)$, respectivamente; o parâmetro $\xi$ é a elasticidade do consumo; e $\rho$ é a taxa de desconto no tempo. As funções de acumulação de capital humano dos empregados e empregadores são as seguintes:

$$
\begin{aligned}
& \stackrel{\circ}{h}=\alpha\left(1-\tau \Omega_{i}\right) f(\varphi, \delta) h^{\alpha} k^{1-\alpha}-c \\
& \stackrel{\circ}{k}=(1-\alpha)\left(1-\tau \Omega_{i}\right) f(\varphi, \delta) h^{\alpha} k^{1-\alpha}-c
\end{aligned}
$$

A solução dos Hamiltonianos para o problema no qual a função objetivo é a equação (6) e as funções de restrições são as equações (8) e (9), respectivamente, de acordo com Chiang (1992), é dada pelas seguintes equações:

$$
\begin{aligned}
& g_{w}=(1 / \xi)\left[\alpha\left(1-\tau \Omega_{i}\right) f(\varphi, \delta)\left(\frac{k}{h}\right)^{1-\alpha}-\rho\right] \\
& g_{k}=(1 / \xi)\left[(1-\alpha)\left(1-\tau \Omega_{i}\right) f(\varphi, \delta)\left(\frac{h}{k}\right)^{1-\alpha}-\rho\right]
\end{aligned}
$$

A taxa de crescimento agregada da economia é dada pela soma das equaçóes (10) e (11). Além disso, as mesmas governam a distribuição de renda entre os empregados e empregadores no tempo. Mantendo as demais variáveis constantes, observa-se que a acumulação de capital humano do empregador representativo influencia positivamente a taxa de crescimento da renda do empregado representativo e vice-versa.

Este modelo implica que, se inicialmente os empregadores tiverem capital humano médio superior ao dos empregados, isto fará com que o crescimento da renda do empregado seja maior que o do empregador $(g(w)>g(k))$; portanto, no longo prazo haverá uma melhora na distribuição de renda entre empregados e empregadores em relação à distribuição inicial. No entanto, devemos ressaltar que o aumento na acumulação de conhecimento dos empregados gera como conseqüência quedas paulatinas na taxa de crescimento de sua renda. Porém, tem como efeito benéfico um aumento na taxa de crescimento da renda dos empregadores. Assim, o sistema gera estímulos para que, por exemplo, a classe dos empregadores incentive a classe dos empregados a acumular conhecimento, uma vez que, indiretamente, estará se beneficiando.

Neste contexto, os investimentos em educação por parte do governo, bem como os desenvolvimentos tecnológicos, são capturados pelas classes sociais através do seu pos- 
sível acesso aos mesmos. O efeito líquido sobre a taxa de crescimento de suas rendas no longo prazo depende das seguintes combinações:

$$
\begin{aligned}
& \frac{\partial g(w)}{\partial \phi}=f_{\phi}^{w}(\phi, \delta) \geq 0 \text { e } \frac{\partial g(w)}{\partial \delta}=f_{\delta}^{w}(\phi, \delta) \geq 0 \\
& \frac{\partial g(k)}{\partial \phi}=f_{\phi}^{k}(\phi, \delta) \geq 0 \text { e } \frac{\partial g(k)}{\partial \delta}=f_{\delta}^{k}(\phi, \delta) \geq 0
\end{aligned}
$$

Observe que as equações (12) e (13) apresentam as derivadas parciais em relação ao nível tecnológico e a infra-estrutura educacional para as respectivas classes. Estas representam o acesso dos empregados e empregadores à tecnologia e à educação, respectivamente. No caso da equação (12), os empregados poderão compensar a redução na sua taxa de crescimento devido à acumulação de maior nível educacional através de maior acesso à tecnologia e/ou educação. $\mathrm{O}$ mesmo acontece com a equação (13), que representa o efeito líquido de tecnologia e infra-estrutura educacional na taxa de crescimento da renda dos empregadores. O maior acesso por uma classe gera como conseqüência maior taxa de crescimento da renda daquela classe em detrimento da outra, causando, portanto, alteração na distribuição de renda.

Assim, o modelo oferece como explicação para a existência da associação positiva entre desigualdade de renda e crescimento econômico um acesso maior à tecnologia e/ou ao sistema educacional por uma determinada classe. Este maior acesso compensa a maior taxação sobre sua renda e os efeitos declinantes da acumulação do nível educacional de sua classe. Em qualquer outro caso, a associação esperada é negativa.

Outro importante aspecto a destacar sobre o modelo é o efeito da acumulação de conhecimento sobre a taxa de crescimento agregada da economia. Se o peso na produtividade for devido ao nível de educação dos empregadores, considerando os demais fatores constantes, então a acumulação de seu conhecimento iria reduzir sua contribuição à taxa de crescimento da produtividade da economia e aumentar a dos empregados. Neste caso, a equação (10) possuiria maior proporção na determinação da taxa de crescimento da produtividade da economia como um todo. Se o oposto for verdadeiro, então a equação (11) possuiria maior proporção na determinação da taxa de crescimento da economia.

A avaliação empírica do crescimento econômico é feita por meio da combinação das equaçôes (10) e (11), em conjunto com as equações (12) e (13). Quando estas equações são combinadas em sua forma reduzida, é obtida a seguinte equação de crescimento agregada: 


$$
g_{y}=g_{w}+g_{k}=f\left(\xi, \tau, h / k, g, \Omega_{i}, A, \rho\right)
$$

Na qual $g_{y}$ é a taxa de crescimento econômico da produtividade, que depende das variáveis já mencionadas.

A extensão da equação acima para os Estados brasileiros pode ser facilmente realizada através da escolha das variáveis apropriadas, que sejam representativas no modelo. A escolha das variáveis, bem como os testes e estimativas econométricas, serão apresentados na próxima seção.

\section{ANÁLISE ECONOMÉTRICA DE PAINEL DE DADOS DOS ESTADOS BRA- SILEIROS}

\subsection{Base de Dados}

A avaliação econométrica do crescimento econômico dos Estados brasileiros, considerando os dados da variável importação de tecnologia, resultou na limitação do período do painel entre 1992 e 1996, em função da disponibilidade de dados. No entanto, segundo Arellano (2003), os trabalhos em painéis para um período como este têm demonstrado que as estimativas dos parâmetros são consistentes e eficientes. $\mathrm{O}$ nosso painel consiste de 26 Estados $(n=26)$ para um período de 5 anos $(\mathrm{T}=5)$, totalizando 130 observações $(\mathrm{N}=\mathrm{nT})$. Outro aspecto importante a ser ressaltado é a categorização do resultado, se este representa o longo ou curto prazo para este painel. Os resultados de trabalhos que utilizam séries temporais são considerados como de curto prazo e os de dados em corte são interpretados como de longo prazo. Segundo Arellano (2003), em painéis onde predominam dados em corte $(\mathrm{N}>\mathrm{T})$, como o caso aqui desenvolvido, a regra é interpretar os resultados como sendo de longo prazo. No entanto, Acemoglu et al. (2005) foram ainda mais longe ao interpretar os efeitos fixos como de longuíssimo prazo, chamando-os de historical development paths. A exceção foi Forbes (2000), que interpretou seus resultados como sendo de médio prazo. Portanto, os resultados a serem reportados são válidos em nossa concepção para o médio e longo prazos, com predominância do último.

Retomando a equação (14), queremos abordar as variáveis a serem utilizadas no processo de estimativa do modelo. A variável dependente a ser utilizada será a taxa de crescimento da produtividade dos trabalhadores dos Estados brasileiros (Tcp). Esta variável resulta da divisão do Produto Interno Bruto dos Estados pelo número de trabalhadores. Neste cômputo, não se incluem os trabalhadores por conta própria, cuja produtividade é de difícil mensuração. Assim, incluímos esta variável na condição de 
controle com o intuito de medir, de forma mais eficiente, sua contribuição para com a produtividade dos Estados. Além disso, esta variável capta os efeitos de regulação e eficiência do mercado de trabalho de cada Estado, conforme sugerido na literatura nacional. Portanto, a contribuição das demais variáveis será líquida da influência destas condiçóes na produtividade. Da mesma forma, incorporamos a variável percentagem de desempregado (na condição de variável controle) para captar dois efeitos apresentados na revisão da literatura nacional. Primeiro, para minimizar as variações na produtividade resultantes apenas da influência relativa de ciclos econômicos em cada Estado, que alteram diretamente o nível de emprego. Segundo, para auxiliar o controle das diferenças de alocação do capital humano entre os Estados, ou seja, as características alocacionais dos mercados de trabalho. A outra variável controle a ser utilizada será uma variável composta, que representa o nível de capital e tecnologia da economia do Estado $\left(Y_{k}\right)$. A construção desta variável requer maiores detalhes, que abordaremos adiante. $\mathrm{O}$ parâmetro $\xi$ será obtido quando da estimativa dos coeficientes das regressões. Conforme a ordem exposta na equação (14), as demais variáveis independentes serão as seguintes: i) a razão entre o nível de escolaridade média dos trabalhadores (Heduc) e dos empregadores (Keduc), cuja nomenclatura a ser utilizada será Reduc, que equivale à variável $h / k$ na equação (14). O sinal será positivo se o fator determinante da taxa de crescimento da produtividade de longo prazo for a acumulação de conhecimento dos empregados e, do contrário, se for dos empregadores; ii) o nível de taxação da economia que é utilizado como investimento em educação, $\delta$, será considerado como o logaritmo da proporção dos investimentos em educação em relação ao PIB de cada Estado (Lieduc). O objetivo é verificar se as variações no investimento em educação $(\delta)$, em relação ao PIB, agem como um imposto realocativo; iii) a distribuição de renda na economia, $\Omega_{i}$, será considerada sob quatro aspectos: o índice de Gini (Gini), a participação na renda dos $40 \%$ mais pobres (Pobre), a participação na renda da classe média ampla (Média), dada pela soma da concentração de renda dos consumidores que estão entre o quinto e oitavo decil ${ }^{4}$ e, finalmente, a participação na renda dos $10 \%$ mais ricos (Rico); e, iv) o nível de tecnologia exógeno da economia será representado pelos gastos com importações de tecnologia em relação ao PIB de cada Estado (Tecim).

A construção da variável nível tecnológico e de capital físico $\left(Y_{k}\right)$ levou em consideração um dos problemas das regressões que explicam as taxas de crescimento econômico, que é a questão de variáveis omitidas. ${ }^{5}$ Para solucionar este problema, procuramos combinar os preceitos teóricos de Lucas (1988) com a sugestão de Temple (1999).

4 Esta definição difere ligeiramente daquela proposta por Easterly (2001a).

5 Para detalhes sobre os problemas de viés da omissão das variáveis, veja Wooldridge (2002). 
A combinação destas duas sugestões consiste em computar os níveis de tecnologia e capital físico de cada Estado através da seguinte equação:

$$
y=A \sum_{\mathrm{i}=1}^{\mathrm{N}}\left(h_{i}^{1-\alpha} k_{i}^{\alpha}\right)(\boldsymbol{h})^{\alpha}
$$

As variáveis da equação (15) são denominadas como: $y$ é o produto por trabalhador de cada empresa no Estado; $N$ é a quantidade de empresas existentes em cada Estado; $k_{i}$ é o estoque de capital físico de cada empresa no mercado; $h_{i}$ é o estoque de capital humano de cada empresa; e, $h=N h_{i}$ é o estoque de capital humano dos Estados. Neste caso, as empresas possuem retornos decrescentes de escala em relação ao seu capital humano, mas quando considerado no agregado a economia do Estado apresenta retornos constantes em relação ao capital humano. Agregando as empresas e considerando que as mesmas são idênticas, como em Lucas (1988), então a economia dos Estados possui a seguinte função de produção por capital humano:

$$
Y_{k}=y / h=A \boldsymbol{k}^{\alpha}
$$

Neste caso, ao se dividir o nível de produto per capita de cada Estado pelo indicador de capital humano agregado, se estaria construindo uma variável proxy que captura os efeitos do nível de tecnologia e do capital físico por trabalhador de cada Estado. Esta variável controle é usada para minimizar os efeitos de variáveis omitidas entre os Estados.

Uma vez estabelecidas as variáveis, a seção a seguir apresenta o modelo econométrico, suas definições, testes e estimativas. A descrição detalhada dos dados para os Estados brasileiros, sua forma de obtenção e fontes foram remetidos ao Apêndice.

\subsection{O Modelo Econométrico}

O modelo econométrico a ser estimado, representado pela equação (14), pressupõe uma relação linear entre as variáveis. A sua forma geral é dada pelo conjunto de equações a seguir:

$$
\begin{aligned}
& y_{i t}=\boldsymbol{x}_{i t} \beta+v_{i t} \\
& v_{i t}=\eta_{i}+u_{i t} \\
& u_{i t}=\sum_{j=1}^{p} \rho_{i j} u_{i, t-j}+\tau_{t}+e_{i t}
\end{aligned}
$$


Sendo $i=1, \ldots, N ; t=1, \ldots, T ;$ e $p$ representa a quantidade de defasagens; a variável $y_{i t}$ é a dependente; o vetor $x_{i t}$ compõe as independentes $(1 \times k) ; \beta$ representa o vetor dos parâmetros a serem estimados $(k \times 1) ; \eta_{\mathrm{i}}$ representa os efeitos fixos ou efeitos específicos não-observados dos Estados; $u_{i t}$ são os erros idiossincráticos; $\left|\rho_{i j}\right|<1$ são os parâmetros que representam a autocorrelação serial, caso existam; e $\tau_{t}$ representa o fator comum entre as seções.

O conjunto de equações (17)-(19) permite representar vários aspectos econométricos que podem estar presentes nos dados. Por exemplo, para o caso em que $\eta_{i}=0$ na equação (18), os Estados não possuiriam efeitos fixos que os fariam diferir entre si. Note-se que a inexistência de autocorrelação na equação (19) pressupõe que $\rho_{i j}=0$. A inexistência de um fator comum a todos Estados, referida aqui também como dependência seccional, implica que $\tau_{t}=0$. Portanto, quando todos estes fatores ocorrem simultaneamente e ainda acontecer de $v_{i t}$ possuir uma distribuição com média zero e variância constante, resultaria em um modelo de efeitos randômicos. No entanto, se $v_{i t}$ não possuir variância constante, então ocorreria o caso de heterocedasticidade, em que a variância dos erros não é a mesma para todas as unidades seccionais.

O modelo de efeitos fixos pressupõe que $\eta_{i} \neq 0$. Este modelo pode apresentar autocorrelação comum a todos os painéis, $\rho_{i j}=\rho$; específica para cada painel, $\rho_{i j}=\rho_{i}$; dependência seccional, $\tau_{t} \neq 0$; e heterocedasticidade; ou ainda, apresentar uma combinação destes fatores. Os testes da seção a seguir visam identificar estes problemas.

\subsubsection{Teste de Estacionariedade}

Um dos grandes problemas das estimativas de modelos econômicos é a questão de regressões espúrias, conforme salienta Jones (1995) e Easterly (2001). As regressões deste gênero normalmente envolvem regredir uma variável estacionária tendo como independentes variáveis não-estacionárias. ${ }^{6} \mathrm{O}$ teste de estacionariedade das variáveis, também denominado raiz unitária, pode ser facilmente visualizado através da seguinte equação:

$$
\Delta y_{i t}=\eta_{i}+\beta_{i} y_{i t-1}+u_{i t}
$$

Na qual $\Delta y_{i t}=y_{i t}-y_{i t}-1$ e $x_{i t}=y_{i t}-1$, em termos da equação (17). O teste de raiz unitária consiste em avaliar a hipótese nula $H_{o}=\beta_{i}=0$. A idéia básica do teste é simples, se $\beta \mathrm{i} \geq 0$, então o coeficiente $\alpha \mathrm{i}$ da regressão $y_{i t}=\alpha_{i} y_{i t-1}+\varepsilon_{i t}$ é maior ou igual a um, ou seja, o passado da variável explica totalmente o seu futuro, portanto as variáveis independentes não influenciam o comportamento da variável no presente. Neste caso, a regressão é espúria, pois não tem sentido o seu relacionamento com outras variáveis.

6 Para maiores detalhes, veja Greene (2000). 
O teste de raiz unitária proposto por Hadri (2000) permite verificar o modelo da equação (20) sob as condições de variâncias idênticas e constantes (homocedásticas) ou diferentes (heterocedásticas). Em nossas estimativas, os resultados sob as duas hipóteses produziram testes estatísticos similares; assim, optamos por reportar somente o caso de heterocedasticidade.

A Tabela 1 demonstra que as variáveis possuem, em geral, probabilidade de estacionariedade. A coluna com a estatística para a tendência foi obtida estimando a equação (20) com uma variável tendência. Neste caso, a análise dos resultados fica relativamente fácil. Tomando como exemplo a variável Tcp, esta possui probabilidade de $86 \%$ de ser estacionária em nível e de $99 \%$ em torno de uma tendência.

A Tabela 1 indica, à exceção das variáveis Tcp, Lieduc e Tecim, que todas as demais variáveis possuem probabilidade de ser estacionárias em torno da tendência, porém abaixo de níveis estatisticamente aceitáveis, cujo limite mínimo seria de 90\%. Estes resultados podem ser uma indicação da existência de outros fatores afetando esta estacionariedade, tais como: heterocedasticidade, dependência seccional ou autocorrelação. Portanto, o teste ideal requer a estimação da equação (20), considerando a validade destas hipóteses em separado.

TABELA 1 - TESTE DE ESTACIONARIEDADE DE HADRI - HIPÓTESE $H_{0}$ : TODAS AS SÉRIES NO PAINEL SÃO ESTACIONÁRIAS

\begin{tabular}{|c|c|c|c|c|c|}
\hline Variável & $\begin{array}{c}\text { Estatística para } \\
\text { Estacionariedade } \\
\text { em Nível }\end{array}$ & $\begin{array}{c}\text { Probabilidade } \\
\text { de } H_{0}\end{array}$ & $\begin{array}{c}\text { Estatística para } \\
\text { Estacionariedade em } \\
\text { Tendência }\end{array}$ & $\begin{array}{l}\text { Probabilidade } \\
\text { de } H_{0}\end{array}$ & $\begin{array}{l}\text { Hipótese da } \\
\text { Variância }\end{array}$ \\
\hline Tсp & $Z_{\mu}=-1,077$ & $P(0,86)$ & $Z_{\mathrm{T}}=-2,47$ & $P(0,99)$ & Hetero \\
\hline$Y_{k}$ & $Z_{\mu}=-2,96$ & $P(0,99)$ & $Z_{T}=-0,80$ & $P(0,79)$ & Hetero \\
\hline Heduc & $Z_{\mu}=4,77$ & $P(0,00)$ & $Z_{T}=-0,26$ & $P(0,60)$ & Hetero \\
\hline Keduc & $Z_{\mu}=4,77$ & $P(0,00)$ & $Z_{T}=-0,50$ & $P(0,69)$ & Hetero \\
\hline Rhk & $Z_{\mu}=0,97$ & $P(0,16)$ & $Z_{T}=-0,34$ & $P(0,63)$ & Hetero \\
\hline Lieduc & $Z_{\mu}=1,22$ & $P(0,00)$ & $Z_{T}=-1,46$ & $P(0,92)$ & Hetero \\
\hline Tecim & $Z_{\mu}=0,51$ & $P(0,31)$ & $Z_{T}=-1,91$ & $P(0,97)$ & Hetero \\
\hline Pcpropria & $Z_{\mu}=1,70$ & $P(0,04)$ & $Z_{T}=-0,79$ & $P(0,78)$ & Hetero \\
\hline Tdes & $Z_{\mu}=0,87$ & $P(0,19)$ & $Z_{T}=0,17$ & $P(0,43)$ & Hetero \\
\hline Gini & $Z_{\mu}=0,66$ & $P(0,25)$ & $Z_{T}=-0,36$ & $P(0,64)$ & Hetero \\
\hline Pobre & $Z_{\mu}=0,72$ & $P(0,23)$ & $Z_{T}=-0,77$ & $P(0,78)$ & Hetero \\
\hline Rico & $Z_{\mu}=0,98$ & $P(0,16)$ & $Z_{T}=-0,05$ & $P(0,48)$ & Hetero \\
\hline Média & $Z_{\mu}=1,22$ & $P(0,11)$ & $Z_{T}=0,32$ & $P(0,37)$ & Hetero \\
\hline
\end{tabular}

Fonte: Calculada pelos autores.

O teste que permite tal flexibilidade foi proposto por Peasaran (2003). O autor estimou os valores críticos das distribuições sob diferentes especificações que consideram as hipóteses citadas. Mais especificamente, o autor estimou as distribuições sob 
autocorrelação serial, dependência seccional e heterocedasticidade. As distribuições estimadas sob estas hipóteses demonstraram ser bastante estáveis para um número de painéis a partir de $10, n \geq 10$, e períodos a partir de $10, T \geq 10$. Como os nossos dados possuem $n=26$ e $T=5$, para os quais o autor não calculou valores específicos, estes exigem que se escolham valores críticos próximos. Os dois valores críticos mais próximos desta combinação pressupóem $N=20$ e $N=30$ para $T=10$. Como os valores críticos para $N=20$ e $T=10$ é o maior dos dois, estes são a opção para a análise. Os valores críticos desta combinação de painel e período são os utilizados para determinar o nível de aceitabilidade dos cálculos obtidos pelos nossos testes. Dessa forma, o modelo econométrico da equação (20) a ser estimado é dado pela seguinte equação:

$$
\Delta y_{i t}=\eta_{i}+\beta_{i} y_{i t-1}+c_{i} \bar{y}_{t-1}+\sum_{j=0}^{p} d_{i j} \Delta \bar{y}_{t-j}+\sum_{j=1}^{p} \delta_{i j} \Delta y_{i t-j}+u_{i t}
$$

Sendo $\bar{y}_{t}=N^{-1} \sum_{j=1}^{N} y_{j t}$, e para $p=j=1$, os termos $\Delta \bar{y}_{t}=\bar{y}_{t}-\bar{y}_{t-1}, \Delta \bar{y}_{t-1}=\bar{y}_{t-1}-\bar{y}_{t-2} \mathrm{e}$ $\Delta y_{i t}=y_{i t}-y_{i t-1}$ auxiliam a eliminar problemas de dependência seccional e autocorrelação. A heterocedasticidade é minimizada por meio de estimativas robustas das variâncias. Note-se que o modelo representado pela equação (2l) é uma extensão para painéis de dados do modelo Dickey-Fuller aumentado. O teste consiste basicamente em corrigir o teste t-estatítico do parâmetro $\beta_{i}$ estimado, a partir da regressão (21). A Tabela 2 apresenta os resultados, conforme as melhores especificações estimadas da equação (21).

Na Tabela 2, a forma de se verificar a aceitabilidade da hipótese, conforme coluna Especificação do Modelo, está em comparar o t-calculado com o valor crítico t-tabelado. A coluna de Probabilidade de $H_{0}$ nos fornece os níveis em que esta é aceitável como verdadeira. Com exceção das varíaveis Heduc e Keduc, todas possuem nível de aceitabilidade da hipótese $H_{0}$ acima de $95 \%$. 
TABELA 2 - TESTE DE ESTACIONARIEDADE DE PEASARAN - Ho: TODAS AS SÉRIES NO PAINEL SÃO ESTACIONÁRIAS

\begin{tabular}{lcccc}
\hline Variável & $\begin{array}{c}\text { Valor Obtido do } \\
\mathrm{t}-\text { Calculado de } \beta_{\mathrm{i}}\end{array}$ & $\begin{array}{c}\text { Valor Crítico do } \\
\mathrm{t} \text {-Tabelado }\end{array}$ & $\begin{array}{c}\text { Probabilidade } \\
\text { de } H_{0}\end{array}$ & $\begin{array}{c}\text { Especificação do } \\
\text { Modelo }\end{array}$ \\
\hline Tcp & $\mathrm{t}=-14,89$ & $\mathrm{t}=-4,97$ & $\mathrm{P}(0,99)$ & Tend e Hetero \\
$Y_{\mathrm{h}}$ & $\mathrm{t}=-2,60$ & $\mathrm{P}(0,99)$ & DS, CS e Hetero \\
Heduc & $\mathrm{t}=-3,80$ & $\mathrm{NE}$ & $\mathrm{P}(0,00)$ & DS, CS e Hetero \\
Keduc & $\mathrm{t}=0,57$ & $\mathrm{NE}$ & $\mathrm{P}(0,00)$ & DS, CS e Hetero \\
Rhk & $\mathrm{t}=-1,22$ & $\mathrm{t}=-2,60$ & $\mathrm{P}(0,99)$ & DS, CS e Hetero \\
Lieduc & $\mathrm{t}=-4,08$ & $\mathrm{t}=-4,35$ & $\mathrm{P}(0,99)$ & Hetero \\
Tecim & $\mathrm{t}=-4,57$ & $\mathrm{t}=-4,35$ & $\mathrm{P}(0,99)$ & Hetero \\
Pcpropria & $\mathrm{t}=-4,54$ & $\mathrm{t}=-3,43$ & $\mathrm{P}(0,95)$ & Hetero \\
Tdes & $\mathrm{t}=-3,45$ & $\mathrm{t}=-2,60$ & $\mathrm{P}(0,99)$ & DS, CS e Hetero \\
Gini & $\mathrm{t}=-3,31$ & $\mathrm{t}=-3,99$ & $\mathrm{P}(0,95)$ & Tend \\
Pobre & $\mathrm{t}=-4,59$ & $\mathrm{t}=-4,97$ & $\mathrm{P}(0,99)$ & Tend \\
Rico & $\mathrm{t}=-5,15$ & $\mathrm{t}=-3,99$ & $\mathrm{P}(0,95)$ & Tend \\
Média & $\mathrm{t}=-4,21$ & $\mathrm{t}=-3,99$ & $\mathrm{P}(0,95)$ & Tend \\
\hline
\end{tabular}

Obs: Tend-Tendência, Hetero-Hetorocedasticidade, DS-Dependência Seccional, CS-Correlação Serial, NE-Não Estacionária e SI-Sem Intercepto.

Fonte: Calculada pelos autores.

A não-estacionariedade das variáveis Heduc e Keduc era esperada, o que nos leva a concluir que estudos que utilizaram os níveis educacionais como a variável proxy para o capital humano podem ter incorrido no problema de regressão espúria. Em nosso caso, especificamente, é provável que este problema não ocorra, pois, de acordo com os testes realizados, a razão entre as duas variáveis, que é representada pela variável Rhk, é estacionária. Em suma, os testes indicam a existência de estacionariedade das variáveis e, portanto, a priori podem ser consideradas nas regressões.

\subsubsection{Teste de Especificação do Modelo}

Um importante fator a ser considerado quando da estimativa do modelo da equação (17) é verificar se o mesmo apresenta efeitos fixos ou randômicos, o que pressupõe que $\eta_{i} \sim I I D\left(0, \sigma_{\eta}^{2}\right)$ e que este é independente de $u_{i t} \sim I I D\left(0, \sigma_{u}^{2}\right)$, em que IID significa independentes e identicamente distribuídos. A rejeição da hipótese de efeitos randômicos implica que os Estados brasileiros possuem características fixas não-observadas que os diferenciam uns dos outros e, portanto, devem ser utilizadas técnicas adequadas. O teste de verificação de efeitos randômicos foi proposto por Breusch e Pagan (1980), baseado no Multiplicador de Lagrange-LM, o qual foi denominado teste dos componentes dos erros. Em conjunto a este teste, deve-se efetuar o da autocorrelação serial, pois esta interfere no resultado do teste de efeitos randômicos. $\mathrm{O}$ teste de autocorrelação serial consiste em verificar se a condição do parâmetro $\rho_{i}=\rho=0$ da equação 
(19) ocorre. Este teste de presença de autocorrelação será efetuado em acordo com o proposto por Baltagi e Li (1995). Para verificar se a presença da autocorrelação está influenciando o teste de efeitos randômicos, será realizado um teste conjunto para ambos. Este último teste foi proposto por Baltagi e Li (1991).

Outro fator importante que pode estar presente nos dados é a heterocedasticidade dos grupos nos painéis. O teste consiste em verificar se $E\left(u_{i t} u_{j t}\right) \neq I \sigma^{2}$ para $t=1, \ldots, T, i \neq j$, em que $I$ é a matriz identidade, ou seja, se as variâncias dos grupos são homocedásticas. O teste baseado na distribuição $\chi^{2}$ (Qui-quadrado) para painéis de dados foi proposto por Greene (2000), e consiste em verificar se os grupos apresentam variâncias idênticas ou não entre os painéis. ${ }^{7}$

Os resultados dos testes propostos encontram-se na Tabela 3. Um teste adicional efetuado, quando da execução dos testes para efeitos randômicos, aparece sob a denominação ALM. Este teste é na verdade o teste LM anterior corrigido para autocorrelação. Ainda de acordo com a Tabela 3, o primeiro teste para efeito randômico LM informa que a probabilidade de os componentes dos erros serem randômicos é de $83 \%$. No entanto, este teste não está corrigido para a autocorrelação, correção esta efetuada com o segundo teste ALM, que informa uma probabilidade de efeitos randômicos de somente 7\%. Este resultado é confirmado quando consideramos os dois testes conjuntos, terceiro teste, em que a probabilidade do teste LM é zero para a hipótese conjunta. O teste para correlação serial possui probabilidade de a hipótese nula ser verdadeira igual a zero. E, por último, o teste de homocedasticidade também rejeita a hipótese nula. Portanto, os testes indicam a presença de efeitos fixos, autocorrelação e heterocedasticidade nos dados.

TABELA 3 - TESTES PARA EFEITOS RANDÔMICOS, CORRELAÇÃO SERIAL E HETEROCEDASTICIDADE

\begin{tabular}{lcc}
\hline Efeitos Randômicos & Resultado do LM & Probabilidade de $H_{0}$ \\
\hline Ho: $\operatorname{Var}\left(\eta_{\mathrm{i}}\right)=0$ & $\mathrm{LM}=-0,98$ & $\mathrm{P}(0,83)$ \\
$\begin{array}{l}\text { Ho: } \operatorname{Var}\left(\eta_{\mathrm{i}}\right)=0 \\
\text { Correlação Serial }\end{array}$ & $\mathrm{ALM}=1,45$ & $\mathrm{P}(0,07)$ \\
$\begin{array}{l}\text { Ho: } \rho=0 \\
\text { Teste Conjunto }\end{array}$ & $\mathrm{LM}=7,99$ & $\mathrm{P}(0,00)$ \\
$\begin{array}{l}\text { Ho: } \operatorname{Var}\left(\eta_{\mathrm{i}}\right)=0 \text { e } \rho=0 \\
\text { Homocedasticidade }\end{array}$ & $\mathrm{LM}=10,08$ & $\mathrm{P}(0,00)$ \\
Ho: $\operatorname{Var}\left(\mathrm{u}_{\mathrm{it}}\right)=\mathrm{I} \sigma^{2}$ & $\chi^{2}(26)=3922$ & $\mathrm{P}(0,00)$ \\
\hline
\end{tabular}

Fonte: Calculada pelos autores.

7 Greene (2000) denominou este teste groupwise heterokesdasticity test. 
Além disso, para confirmar se devemos considerar um modelo de efeitos fixos e/ou randômicos, aplicamos um teste complementar, baseado em Hausman (1978), de especificação do modelo. Este teste consiste em comparar os coeficientes das regressões sob efeitos fixos e randômicos. O resultado do teste Qui-quadrado comparando os coeficientes foi $\chi(5)=8,90$, com uma probabilidade de $\mathrm{P}(0,06)$. Portanto, esta probabilidade não é alta o suficiente para rejeitar o modelo de efeitos fixos. Cabe ressaltar que os resultados deste teste podem estar sendo influenciados pela presença de autocorrelação.

Quanto à confirmação da presença de autocorrrelação, especificamente, efetuamos um teste adicional, proposto por Wooldridge (2002), para a hipótese de que $\rho=0$. O teste $F(1,25)=0,09$ deste parâmetro possui uma probabilidade de $76 \%, P(0,76)$, de aceitabilidade da hipótese nula, de não existência de autocorrelação. Este resultado contradiz o apresentado na Tabela 3. A explicação é que, neste teste, corrigimos a heterocedasticidade e os efeitos fixos. Portanto, a combinação destes dois resultados opostos pode ser um indicador da existência de autocorrelação específica para cada painel e não única a todos os painéis, conforme os testes implementados.

Em geral, nossos testes sugerem um modelo de efeitos fixos como o mais plausível, corrigidos para autocorrelação específica de painéis de dados e heterocedasticidade. Outro elemento importante é considerar o potencial de dependência seccional dos painéis.

\subsection{Estimativas do Modelo Econométrico}

\subsubsection{O Modelo Básico}

Neste modelo básico, a equação (17) é estimada considerando como variável dependente Tcp, as variáveis independentes como Rhk e Lieduc, e as variáveis controle Pcpropria e Tdes. As regressões com painel de dados dos Estados brasileiros foram efetuadas sob diferentes especificações. A primeira coluna da Tabela 4 é um modelo de efeitos randômicos e a segunda coluna, um modelo de efeitos fixos, ambos obtidos usando o método de mínimos quadrados para painel de dados. Estas duas especificações serão utilizadas como base, pois não corrigem autocorrelação, heterocedasticidade e dependência seccional. Estas hipóteses serão consideradas nos modelos a serem estimados a posteriori, o que permite comparar as estimativas e identificar os fatores que estão influenciando os resultados.

Ainda com relação à Tabela 4, a variável Reduc, que é a razão do nível de educação dos empregados-empregadores, se apresenta como significante e positiva em ambos 
os modelos. A variável investimento em educação (Lieduc) é significante nos dois modelos e o sinal negativo indica que o mesmo age como um imposto e, portanto, reduz a taxa de crescimento da produtividade. No entanto, o seu efeito é compensado pela variável Reduc. Ainda sobre os resultados desta tabela, é importante analisar o sinal positivo da variável Reduc em consonância com as equações (10) e (11). Este sinal positivo significa que os empregados estão acumulando conhecimento a uma taxa superior à dos empregadores, portanto $h / k$ está aumentando. Como a taxa de crescimento da produtividade da economia é a soma destas duas taxas, isto significa que os aumentos verificados nesta taxa são decorrentes do aumento do conhecimento dos empregados vis-à-vis empregadores. Mas, de acordo com a equação (11), isto indica ainda que a taxa de crescimento da renda dos empregadores $g_{k}$ está se beneficiando, pois está aumentando com os aumentos desta razão. Já a equação (10) prevê que a influência do aumento desta razão irá reduzir a taxa de crescimento da renda dos empregados no longo prazo. A conseqüência esperada é um aumento na distribuição de renda e, portanto, maior taxação dos empregadores, caso estes detenham maior nível de renda.
TABELA 4 - MODELO BÁSICO - VARIÁVEL DEPENDENTE: TAXA DE CRESCIMENTO DA PRODUTIVIDADE DOS ESTADOS BRASILEIROS (TCP)

\begin{tabular}{lll}
\hline Variáveis & ER & EF \\
\hline \multirow{2}{*}{ Reduc } & 0,139 & 0,233 \\
& $(0,064)^{* *}$ & $(0,102)^{* *}$ \\
Lieduc & $-0,036$ & $-0,063$ \\
& $(0,015)^{* *}$ & $(0,034)^{* * *}$ \\
Pcpropria & 0,198 & 0,330 \\
& $(0,149)$ & $(0,645)$ \\
Tdes & 0,287 & 1,717 \\
& $(0,92)$ & $(0,613)^{*}$ \\
Constante & $-0,254$ & $-0,542$ \\
N & $(0,108)^{* *}$ & $(0,227)^{*}$ \\
\hline
\end{tabular}

Obs: ${ }^{*}$ significante a $1 \%,{ }^{* *}$ significante a $5 \% \mathrm{e}^{* *}$ significante a $10 \%$. ER-Efeitos Randômicos, EFEfeitos Fixos e N=Número de observaçốes.

Fonte: Calculada pelos autores.

Quanto às outras variáveis, a percentagem de trabalhadores por conta própria (Pcpropria) não aparece como significativa, enquanto a taxa de desemprego dos Estados (Tdes) se mostrou significante somente no modelo de efeitos fixos. Como ambas as variáveis são utilizadas como controle, remetemos suas análises para a próxima seção que estima um modelo mais completo. 


\subsubsection{Os Efeitos da Desigualdade de Renda}

Nesta seção, estimamos um modelo completo, considerando os efeitos da desigualdade de renda sobre a taxa de crescimento da produtividade dos trabalhadores de longo prazo. Nesta primeira estimativa, visando capturar fatores específicos dos Estados, consideramos uma sugestão de Peasaram (2003). Especificamente para o caso aqui estudado, utilizamos as variáveis de distribuição de renda com o intuito de capturar efeitos específicos dos Estados, através da seguinte representação:

$$
\Delta y_{i t}=\beta \Delta y_{i t-1}+e_{i t}
$$

Basicamente, estima-se o vetor $\beta$ por meio de mínimos quadrados sobre os dados em corte e temporais (pooled), e os resíduos da equação $\Delta y_{i t}-\hat{\beta} \Delta y_{i t-1}=\hat{e}_{i t}$ são utilizados como proxies para as diferenças remanescentes entre os Estados. Estes resíduos aparecem na regressão como as variáveis Pobre, Média, Rico e Gini, adicionadas da letra $r$.

Este modelo, visando minimizar os efeitos fixos e de dependência seccional, além das variáveis de controle do modelo básico, contém as seguintes variáveis indicadoras (dummies) adicionais: Sul, que representa os Estados do Paraná, Santa Catarina e Rio Grande do Sul; Sudeste, que corresponde aos Estados de São Paulo, Rio de Janeiro, Espírito Santo e Minas Gerais; Centro-Oeste, que compreende os Estados de Goiás, Mato Grosso, Mato Grosso do Sul e o Distrito Federal; ${ }^{8}$ Norte, que engloba os Estados do Acre, Amazonas, Amapá, Pará, Rondônia, Roraima e Sergipe; e, Nordeste, que considera os Estados de Alagoas, Bahia, Ceará, Paraíba, Pernambuco, Piauí e Rio Grande do Norte em sua definição. Para mensurar as regiões de forma relativa, a região Sudeste foi suprimida por escolha, assim todas as estimativas serão relativas a esta região. Além disso, as variáveis indicadoras são interagidas com a variável ano.

Assim, essa especificação é uma forma de admitirmos que os Estados enquanto pertencentes a uma mesma região possuem características similares entre si, conforme resultado de Mossi et alli (2003). Assim, a sugestão técnica de Hsiao (2003) foi seguida, de tal forma que possamos capturar as variáveis omitidas remanescentes dos Estados através da diferença de suas regiões e das diferenças das desigualdades de rendas, mas mantendo o modelo como tendo origem randômica. A razão para este comportamento está na inferência a ser feita. As explicações de Hsiao (2003) são claras a este respeito. Os modelos de efeitos fixos são aqueles em que o investigador faz inferências condicionais aos efeitos que estão na amostra, enquanto os modelos de efeitos randômicos permitem que o investigador faça inferências marginais a respeito

8 Tocantins foi considerado juntamente com o Estado de Goiás. 
para a população. Assim, os resultados do modelo a seguir podem ser interpretados de forma mais abrangente.

Na estimativa do modelo econométrico da equação (17) foram levados em consideração os resultados dos testes que indicaram a presença potencial de autocorrelação e heterocedasticidade. Para corrigir estes efeitos, foi utilizado o método de Mínimos Quadrados Generalizados Factíveis (MQGF), conforme apresentado em Judge et alli (1985). Este método consiste em estimar os coeficientes da regressão através do método de Mínimos Quadrados Generalizados da seguinte forma:

$$
\hat{\beta}=\left(\sum_{i=1}^{N} x_{i}^{\prime} \Theta^{-1} x_{i}\right)\left(\sum_{i=1}^{N} x_{i}^{\prime} \Theta^{-1} y_{i}\right)
$$

$\mathrm{Na}$ qual a matriz de pesos $\Theta$ é estimada tendo por base a seguinte matriz:

$$
\hat{\Theta}=N^{-1} \sum_{i=1}^{N} \hat{v_{i}} \hat{v_{i}^{\prime}}
$$

Sendo $v_{i}$ o vetor de erros do primeiro estágio da regressão, conforme equação (17). Esta matriz de variâncias e co-variâncias usada como matriz peso tem por objetivo minimizar os efeitos da heterocedasticidade e da autocorrelação. A estimativa considera que as variáveis independentes possuem exogeneidade contemporânea em relação à dependente, ou seja, no presente são as variáveis níveis de educação, desigualdade de renda, investimento em educação, importação de tecnologia e nível tecnológico que afetam o crescimento da produtividade.

Os resultados das estimativas estão na Tabela 5 a seguir. Na primeira coluna, temos a regressão básica sem considerar as variáveis de desigualdade de renda e, portanto, nos serve para comparar com os demais resultados. Nesta coluna, a variável razão dos níveis educacionais dos empregados-empregadores (Reduc) é altamente significativa, indicando que o efeito de acumulação de nível de conhecimento dos empregados vis-à-vis dos empregadores é benéfico para um aumento na taxa de crescimento da produtividade dos Estados. A variável investimento em educação (Lieduc) aparece com o sinal negativo, o que indica o seu efeito realocativo de insumos para o setor produtor de capital humano. A importação de tecnologia (Tecim) não se apresenta significante. As variáveis controle, percentagem de trabalhadores por conta própria (Pcpropria) e taxa de desemprego (Tdes) são significativas nesta especificação. Estas parecem indicar que os mercados de trabalho dos Estados possuem interferência direta no crescimento da produtividade, conforme ressaltado por Barros e Mendonça (1996) e Azzoni e Servo (2002). Em nossa avaliação, a primeira (Pcpropria) captura sua contribuição direta para a produtividade dos Estados, enquanto a segunda (Tdes) 
reflete a eficiência de alocação dos recursos humanos nos Estados para o longo prazo. O resultado como um todo corrobora a tese de que a rigidez nas características dos mercados de trabalho afeta a taxa de crescimento da produtividade. Como o nível de escolaridade dos trabalhadores influencia a produtividade de forma positiva, parece haver uma forte indicação da preferência do mercado por capital humano sem oferta correspondente. No entanto, deve-se realizar um estudo criterioso sobre o que precisamente estas variáveis podem estar capturando.

O nível de tecnologia dos Estados $\left(Y_{k}\right)$ é significante, com sinal negativo. Isto significa que em Estados onde o nível tecnológico é maior, a produtividade cresce a uma taxa menor. Este resultado é interessante, pois quando associado com a variável tempo (Ano), que é negativa e significante, pode refletir uma queda na taxa de crescimento da produtividade com maior grau de desenvolvimento econômico no tempo.

A interação da variável tempo com as regiões nos informa que a região Sul está crescendo de forma diferenciada e superior em relação à região Sudeste. Por outro lado a região Norte apresenta taxas de crescimento da produtividade inferior à região Sudeste. Como os coeficientes das demais regiões não são significativos, pode-se inferir que estas apresentam tendência de crescimento equivalente à da região Sudeste.

A coluna (2) da Tabela 5 inclui as diferenças remanescentes entre os Estados em relação à variável de desigualdade de renda. Note-se que esta variável representa a concentração de renda dos $40 \%$ mais pobres. O seu efeito significativo e positivo indica que políticas que aumentam e tornam a participação dos $40 \%$ mais pobres, mais uniforme, na renda total resulta em maior taxa de crescimento da produtividade do Estado.

Na coluna (3) da referida tabela, observa-se que políticas que incentivam maior participação da classe média na renda dos Estados parecem não ter efeito sobre o crescimento da produtividade. Nas colunas (4) e (5), tanto a participação dos $10 \%$ mais ricos, como da desigualdade como um todo, representada pelo índice de Gini, demonstram que os seus efeitos são claramente negativos para o crescimento da produtividade. Estes resultados indicam uma associação negativa entre desigualdade de renda e crescimento da produtividade. Portanto, a previsão do modelo de que a associação positiva ocorre somente se uma das classes estiver acessando tecnologia e educação em detrimento da outra parece não se verificar no caso dos Estados brasileiros. 
TABELA 5 - DIFERENÇAS REGIONAIS - EFEITOS RANDÔMICOS - MÉTODO MQGF - VARIÁVEL DEPENDENTE: TAXA DE CRESCIMENTO DA PRODUTIVIDADE DOS ESTADOS (TCP)

\begin{tabular}{|c|c|c|c|c|c|}
\hline Variáveis & (1) & (2) & (3) & (4) & (5) \\
\hline Reduc & $\begin{array}{c}0,196 \\
(0,03)^{*}\end{array}$ & $\begin{array}{c}0,150 \\
(0,03)^{*}\end{array}$ & $\begin{array}{c}0,188 \\
(0,003)^{*}\end{array}$ & $\begin{array}{c}0,239 \\
(0,003)^{*}\end{array}$ & $\begin{array}{c}0,155 \\
(0,03)^{*}\end{array}$ \\
\hline Lieduc & $\begin{array}{l}-0,0017 \\
(0,006)^{*}\end{array}$ & $\begin{array}{l}-0,0017 \\
(0,006)^{*}\end{array}$ & $\begin{array}{c}0,009 \\
(0,008)\end{array}$ & $\begin{array}{l}-0,012 \\
(0,010)\end{array}$ & $\begin{array}{l}-0,020 \\
(0,007)^{*}\end{array}$ \\
\hline Tecim & $\begin{array}{c}0,0007 \\
(0,003)\end{array}$ & $\begin{array}{c}0,0008 \\
(0,003)\end{array}$ & $\begin{array}{c}0,0004 \\
(0,003)\end{array}$ & $\begin{array}{c}0,005 \\
(0,003)\end{array}$ & $\begin{array}{l}-0,0011 \\
(0,002)\end{array}$ \\
\hline Pcpropria & $\begin{array}{c}0,466 \\
(0,088)^{*}\end{array}$ & $\begin{array}{c}0,303 \\
(0,061)^{*}\end{array}$ & $\begin{array}{c}0,546 \\
(0,038)^{*}\end{array}$ & $\begin{array}{c}0,739 \\
(0,089)^{*}\end{array}$ & $\begin{array}{c}0,365 \\
(0,070)^{*}\end{array}$ \\
\hline Tdes & $\begin{array}{c}0,971 \\
(0,138)^{*}\end{array}$ & $\begin{array}{c}0,354 \\
(0,099)^{*}\end{array}$ & $\begin{array}{c}0,527 \\
(0,113)^{*}\end{array}$ & $\begin{array}{c}1,119 \\
(0,168)^{*}\end{array}$ & $\begin{array}{c}0,350 \\
(0,121)^{*}\end{array}$ \\
\hline$Y_{k}$ & $\begin{array}{l}-2,7 \mathrm{e}-06 \\
(1,4 \mathrm{e}-06)^{\star *}\end{array}$ & $\begin{array}{l}-1,5 \mathrm{e}-06 \\
(9,8 \mathrm{e}-07)^{\star \star *}\end{array}$ & $\begin{array}{l}-4,1 \mathrm{e}-07 \\
(7,6 e-07)\end{array}$ & $\begin{array}{l}-4,8 \mathrm{e}-06 \\
(1,4 \mathrm{e}-06)^{*}\end{array}$ & $\begin{array}{l}-1,8 \mathrm{e}-06 \\
(9,5 \mathrm{e}-07)^{* *}\end{array}$ \\
\hline Ano & $\begin{array}{l}-0,00013 \\
(0,00003)^{*}\end{array}$ & $\begin{array}{l}-0,000098 \\
(0,00002)^{*}\end{array}$ & $\begin{array}{l}-0,00013 \\
(6,2 \mathrm{e}-06)^{*}\end{array}$ & $\begin{array}{l}-0,00020 \\
(0,00002)^{*}\end{array}$ & $\begin{array}{l}-0,00010 \\
(0,00002)^{*}\end{array}$ \\
\hline Ano*Sul & $\begin{array}{r}0,000018 \\
(5,2 \mathrm{e}-06)^{*}\end{array}$ & $\begin{array}{l}-5,2 \mathrm{e}-06 \\
(3,7 \mathrm{e}-06)\end{array}$ & $\begin{array}{l}-1,9 \mathrm{e}-06 \\
(3,4 \mathrm{e}-06)\end{array}$ & $\begin{array}{r}0,000013 \\
(4,8 \mathrm{e}-06)^{*}\end{array}$ & $\begin{array}{l}-7,7 e-06 \\
(3,8 \mathrm{e}-06)^{* *}\end{array}$ \\
\hline Ano*Centro-Oeste & $\begin{array}{c}5,7 e-06 \\
(6,0 e-06)\end{array}$ & $\begin{array}{l}-8,4 \mathrm{e}-06 \\
(4,0 \mathrm{e}-06)^{\star *}\end{array}$ & $\begin{array}{l}-4,2 e-06 \\
(6,2 e-06)\end{array}$ & $\begin{array}{c}9,5 \mathrm{e}-06 \\
(4,4 \mathrm{e}-06)^{\star *}\end{array}$ & $\begin{array}{l}-0,000010 \\
(4,1 \mathrm{e}-06)^{*}\end{array}$ \\
\hline Ano*Nordeste & $\begin{array}{l}-3,7 e-06 \\
(4,7 e-06)\end{array}$ & $\begin{array}{l}-0,00001 \\
\left(5,7 e-06^{\star *}\right.\end{array}$ & $\begin{array}{l}-0,000013 \\
(6,2 \mathrm{e}-06)^{\star *}\end{array}$ & $\begin{array}{l}-0,000015 \\
(5,7 e-06)^{*}\end{array}$ & $\begin{array}{l}-0,000013 \\
(6,2 \mathrm{e}-06)\end{array}$ \\
\hline Ano*Norte & $\begin{array}{l}-0,000023 \\
(5,8 \mathrm{e}-06)^{*}\end{array}$ & $\begin{array}{l}-0,00003 \\
(6,9 e-06)^{*}\end{array}$ & $\begin{array}{l}-0,00004 \\
(6,1 \mathrm{e}-06)^{*}\end{array}$ & $\begin{array}{r}0,000045 \\
(5,0 \mathrm{e}-06)^{*}\end{array}$ & $\begin{array}{c}-0,000038 \\
(, 67 e-06)^{*}\end{array}$ \\
\hline Pobrer & & $\begin{array}{c}1,779 \\
(0,330)^{*}\end{array}$ & & & \\
\hline Médiar & & & $\begin{array}{l}-0,011 \\
(0,20)\end{array}$ & & \\
\hline Ricor & & & & $\begin{array}{l}-4,931 \\
(1,188)^{*}\end{array}$ & \\
\hline Ginir & & & & & $\begin{array}{l}-0,606 \\
(0,159)^{*}\end{array}$ \\
\hline $\mathrm{N}$ & 78 & 78 & 78 & 78 & 78 \\
\hline $\mathrm{R}^{2}$ & - & - & - & - & - \\
\hline
\end{tabular}

Obs: ${ }^{*}$ significante a $1 \% ;{ }^{* *}$ significante a $5 \% \mathrm{e}^{* * *}$ significante a $10 \%$; N é o número de observações. Fonte: Calculada pelos autores. 


\subsubsection{Considerando os Efeitos Fixos dos Estados}

Nesta seção é estimado um modelo que considera os efeitos fixos não-observados dos Estados. Na estimativa do modelo de efeitos fixos, é preciso considerar os problemas da autocorrelação e da heterocedasticidade mencionados anteriormente. Neste caso, a melhor estratégia é utilizar a forma sugerida por Kiefer (1980). De acordo com o autor, com relação à equação (17), deve-se proceder da seguinte forma. Primeiro, define-se:

$$
\bar{y}_{i}=\overline{\mathbf{x}}_{i} \beta+\bar{u}_{i}
$$

Sendo $\bar{y}_{i}=T^{-1} \sum_{i=1}^{T} y_{i t}, \overline{\mathbf{x}}_{i}=T^{-1} \sum_{i=1}^{T} \mathbf{x}_{i t}$ e $\overline{\mathrm{u}}_{i}=T^{-1} \sum_{i=1}^{T} \mathrm{u}_{i t}$, sendo que $i=1, \ldots, n$ e $T=1, \ldots, T$.

Subtraindo a equação (25) da equação (17), obtém-se o modelo de efeitos fixos a ser estimado, ou seja:

$$
y_{i t}-\overline{y_{i}}=\left(\mathbf{x}_{\mathrm{it}}-\mathbf{x}_{i}\right) \beta+\left(u_{i t}-\overline{u_{i}}\right)
$$

Então, a equação (26) é estimada por meio do método de Mínimos Quadrados Generalizados Factíveis, conforme equações (23) e (24). De acordo com a especificação da equação (26), a variável $\eta_{i}$ é eliminada pelas diferenças. Portanto, a correlação com as variáveis independentes é eliminada ou minimizada de forma a não interferir nos resultados.

Os resultados do modelo estão na Tabela 6, em que os nomes das variáveis aparecem seguidos do sufixo ef para efeitos fixos. Conforme Tabela 6, sob o modelo de efeitos fixos, todas as variáveis passaram a ser significantes. As estimativas também demonstram ser robustas em relação às diversas definições da variável desigualdade de renda, pois os coeficientes obtidos são estatisticamente equivalentes em todas as especificações. Ou seja, as variáveis razão do nível de conhecimento dos empregadosempregadores (Reducef), logaritmo do investimento em educação como proporção do PIB (Lieducef), tecnologia importada (Tecimef), percentagem dos trabalhadores por conta própria (Pcpropriaef), nível de capital físico e tecnologia $\left(\mathrm{Y}_{\mathrm{k}} \mathrm{ef}\right)$, participação na renda dos $40 \%$ mais pobres (Pobreef), participação na renda da classe média (Médiaef), participação na renda dos $10 \%$ mais ricos (ricoef) e do índice de desigualdade de renda Gini (Ginief) demonstram ter significância estatística na taxa de crescimento de produtividade dos Estados.

No conjunto das regressões apresentadas na Tabela 6, a variável ano foi suprimida, pois a sua diferença no tempo é uma constante. A constante da regressão é significante 
e positiva, refletindo a taxa média do crescimento da produtividade de longo prazo associada a efeitos não-observados no modelo. Nesta especificação, procuramos manter as variáveis de tendência das regiões, visando capturar alterações ainda remanescentes, que pudessem estar associadas às regióes e não aos Estados.

TABELA 6 - ESTADOS - EFEITOS FIXOS - VARIÁVEL DEPENDENTE: TAXA DE CRESCIMENTO DA PRODUTIVIDADE DOS ESTADOS (TCP)

\begin{tabular}{|c|c|c|c|c|c|}
\hline Variáveis & (1) & (2) & (3) & (4) & (5) \\
\hline Reducef & $\begin{array}{c}0,241 \\
(0,018)^{*}\end{array}$ & $\begin{array}{c}0,241 \\
(0,020)^{*}\end{array}$ & $\begin{array}{c}0,242 \\
(0,029)^{*}\end{array}$ & $\begin{array}{c}0,240 \\
(0,019)^{*}\end{array}$ & $\begin{array}{c}0,238 \\
(0,193)^{*}\end{array}$ \\
\hline Lieducef & $\begin{array}{l}-0,022 \\
(0,004)^{\star}\end{array}$ & $\begin{array}{l}-0,022 \\
(0,005)^{\star}\end{array}$ & $\begin{array}{l}-0,026 \\
(0,005)^{\star}\end{array}$ & $\begin{array}{l}-0,027 \\
(0,005)^{\star}\end{array}$ & $\begin{array}{l}-0,025 \\
(0,006)^{*}\end{array}$ \\
\hline Tecimef & $\begin{array}{c}0,004 \\
(0,003)\end{array}$ & $\begin{array}{c}0,009 \\
(0,003)^{*}\end{array}$ & $\begin{array}{l}0,006 \\
(0,003)^{\star * *}\end{array}$ & $\begin{array}{c}0,007 \\
(0,003)^{\star *}\end{array}$ & $\begin{array}{c}0,008 \\
(0,003)^{*}\end{array}$ \\
\hline Pcpropriaef & $\begin{array}{c}0,346 \\
(0,053)^{*}\end{array}$ & $\begin{array}{c}0,422 \\
(0,048)^{*}\end{array}$ & $\begin{array}{c}0,373 \\
(0,062)^{*}\end{array}$ & $\begin{array}{c}0,382 \\
(0,058)^{*}\end{array}$ & $\begin{array}{c}0,346 \\
(0,066)^{*}\end{array}$ \\
\hline Tdesef & $\begin{array}{l}1,00 \\
(0,108)^{*}\end{array}$ & $\begin{array}{c}1,11 \\
(0,08)^{\star}\end{array}$ & $\begin{array}{c}1,05 \\
(0,105)^{*}\end{array}$ & $\begin{array}{l}1,06 \\
(0,097)^{*}\end{array}$ & $\begin{array}{l}1,06 \\
(0,108)^{\star}\end{array}$ \\
\hline$Y_{k} e f$ & $\begin{array}{l}-0,000012 \\
(1,2 \mathrm{e}-06)^{*}\end{array}$ & $\begin{array}{l}-0,000011 \\
(5,5 \mathrm{e}-07)^{*}\end{array}$ & $\begin{array}{l}-0,000012 \\
(9,9 \mathrm{e}-07)^{\star}\end{array}$ & $\begin{array}{l}-0,000011 \\
(8,9 \mathrm{e}-06)^{*}\end{array}$ & $\begin{array}{l}-0,000011 \\
(1,0 \mathrm{e}-06)^{*}\end{array}$ \\
\hline Ano*Sul & $\begin{array}{r}0,000017 \\
(5,8 \mathrm{e}-06)^{*}\end{array}$ & $\begin{array}{r}0,000018 \\
(6,6 \mathrm{e}-06)^{*}\end{array}$ & $\begin{array}{c}0,000017 \\
(6,0 \mathrm{e}-06)^{*}\end{array}$ & $\begin{array}{r}0,000017 \\
(6,0 \mathrm{e}-06)^{*}\end{array}$ & $\begin{array}{r}0,000018 \\
(6,1 \mathrm{e}-06)^{*}\end{array}$ \\
\hline Ano*Centro-Oeste & $\begin{array}{r}0,000011 \\
(4,1 \mathrm{e}-06)^{*}\end{array}$ & $\begin{array}{r}0,000016 \\
(4,6 \mathrm{e}-06)^{*}\end{array}$ & $\begin{array}{r}0,000011 \\
(3,9 \mathrm{e}-06)^{*}\end{array}$ & $\begin{array}{r}0,000013 \\
(3,7 \mathrm{e}-06)^{*}\end{array}$ & $\begin{array}{r}0,000016 \\
(4,4 \mathrm{e}-06)^{*}\end{array}$ \\
\hline Ano*Nordeste & $\begin{array}{c}-7,7 \mathrm{e}-06 \\
(4,5 \mathrm{e}-06)^{\star * *}\end{array}$ & $\begin{array}{c}2,1 \mathrm{e}-06 \\
(6,4 \mathrm{e}-06)\end{array}$ & $\begin{array}{l}-3,1 e-06 \\
(4,8 e-06)\end{array}$ & $\begin{array}{c}9,6 \mathrm{e}-07 \\
(5,1 \mathrm{e}-06)\end{array}$ & $\begin{array}{c}2,0 \mathrm{e}-06 \\
(6,0 \mathrm{e}-06)\end{array}$ \\
\hline Ano*Norte & $\begin{array}{l}-0,000029 \\
(4,8 \mathrm{e}-06)^{*}\end{array}$ & $\begin{array}{l}-0,000027 \\
(4,3 \mathrm{e}-06)^{*}\end{array}$ & $\begin{array}{l}-0,000026 \\
(5,1 \mathrm{e}-06)^{*}\end{array}$ & $\begin{array}{l}-0,00025 \\
(4,9 \mathrm{e}-06)^{*}\end{array}$ & $\begin{array}{l}-0,000023 \\
(5,4 \mathrm{e}-06)^{*}\end{array}$ \\
\hline Constante & $\begin{array}{c}0,011 \\
(0,007)^{*}\end{array}$ & $\begin{array}{c}0,006 \\
(0,008)\end{array}$ & $\begin{array}{c}0,011 \\
(0,007)^{*}\end{array}$ & & $\begin{array}{c}0,005 \\
(0,008)^{*}\end{array}$ \\
\hline Pobreef & & $\begin{array}{c}0,853 \\
(0,366)^{* *}\end{array}$ & & & \\
\hline Médiaef & & & $\begin{array}{c}0,137 \\
(0,063)^{\star *}\end{array}$ & & \\
\hline Ricoef & & & & $\begin{array}{l}-0,155 \\
(0,058)^{\star}\end{array}$ & \\
\hline Ginief & & & & & $\begin{array}{l}-0,266 \\
(0,098)^{*}\end{array}$ \\
\hline $\mathrm{N}$ & 104 & 104 & 104 & 104 & 104 \\
\hline$R^{2}$ & - & - & - & - & - \\
\hline
\end{tabular}

Obs: ${ }^{*}$ significante a $1 \% ;{ }^{* *}$ significante a $5 \% \mathrm{e}^{* * *}$ significante a $10 \%$; $\mathrm{N}$ é o número de observações. Fonte: Calculado pelos autores. 
Além disso, a razão nível educacional dos empregados-empregadores continua sendo um dos fatores determinantes dos aumentos na taxa de crescimento da produtividade. Entre as demais variáveis observamos três destaques: o primeiro é a confirmação de que os investimentos em educação dos Estados têm influência negativa devido ao efeito realocacional de insumos do setor produtivo para o setor educacional. Esta pode ser uma explicação para o fato de as políticas educacionais serem de difícil implementação, já que seus resultados somente aparecem no longo prazo, através da melhoria dos níveis educacionais de ambas as classes.

O segundo destaque refere-se às tendências das regiões. As regiões Sul e Centro-Oeste confirmam o fato de suas tendências estarem crescendo acima da região Sudeste, enquanto a região Norte apresenta tendência de crescimento inferior relativamente à região Sudeste.

O terceiro destaque é a variável importação de tecnologia, Tecimef. Esta aparece como significante nas especificações em que consideramos as variáveis de distribuição de renda, nas colunas (2) - (5). Este resultado é importante, pois demonstra o papel exercido pela inovação externa no aumento da produtividade dos Estados brasileiros.

Um outro aspecto desta especificação com efeitos fixos e que podemos inferir de forma indireta é com relação à convergência de produtividade entre os Estados. ${ }^{9} \mathrm{O}$ coeficiente negativo e significante do nível de capital físico e de tecnologia dos Estados $\left(Y_{k} e f\right)$ pode ser uma indicação de convergência ao longo do tempo do seu nível de produtividade em relação a este grau de desenvolvimento. Ou seja, quanto maior for o nível de capital físico e de tecnologia, mais desenvolvida será a economia e menor será a taxa de crescimento de sua produtividade de longo prazo. Mas esta condição de convergência somente será válida se as demais variáveis permanecerem constantes. Por exemplo, maior acumulação de capital humano dos empregados em relação aos empregadores, conforme resultado obtido, pode eliminar completamente a questão de convergência.

\subsubsection{Educação e Crescimento Econômico}

Esta seção explora o papel da educação e dos investimentos em educação sobre a taxa de crescimento da produtividade de longo prazo. O intuito é verificar se os efeitos dos níveis educacionais e dos investimentos são não-lineares e, assim, confirmar ou não a existência de um nível ótimo entre a razão do nível de educação dos empregadosempregadores e a taxa de crescimento da produtividade dos Estados brasileiros.

9 A forma é indireta, pois o teste de convergência exige regressões mais apropriadas. As referências básicas na área de convergência dos Estados brasileiros são Ferreira e Ellery (1996), Zini Jr. (1998) e Azzoni (2001). 
O modelo a ser estimado é o de efeitos fixos, composto pelas equações (17) a (19), portanto equivalente ao da seção anterior com correção para autocorrelação e heterocedasticidade de painéis de dados, com a ressalva que adicionamos a variável Reduc2, que representa a variável Reduc ao quadrado. Como a variável Reduc é inferior a um, o seu valor ao quadrado captura os efeitos de uma razão menor entre o nível de educação dos empregados e empregadores. Neste caso, a variável Reduc capta o efeito de um aumento nesta razão e seus benefícios à taxa de crescimento da produtividade dos Estados brasileiros no longo prazo. Os resultados econométricos encontram-se na Tabela 7.

Sob esta especificação, a variável Reduc2 é significante somente quando associada às variáveis Pobreef, Ricoef e Ginief. Portanto, os resultados da Tabela 7 levam-nos a inferir que os Estados cujos benefícios de aumento no nível de educação dos empregados vis-à-vis os empregadores são aqueles nos quais há um menor nível de concentração de renda entre os $40 \%$ mais pobres (coluna (2) da Tabela 7); os que têm maior concentração de renda entre os 10\% mais ricos (coluna (4)); e, aqueles Estados onde o nível geral de desigualdade de renda é maior (coluna (5)). Estes resultados são compatíveis com a literatura revisada, que indica a necessidade de uma política educacional mais intensa, que beneficie acima de tudo os mais pobres, pois reduziria a desigualdade de renda e auxiliaria o crescimento da produtividade.

O nosso resultado acrescenta que esta política seria mais eficiente se fossem os trabalhadores vis-à-vis os empregadores os beneficiados, em função da ligação existente entre a razão de seus conhecimentos e o crescimento da produtividade. Em suma, o que o nosso modelo estimado faz é demonstrar os benefícios desta política para o binômio redução da desigualdade renda e crescimento da produtividade da economia dos Estados no longo prazo.

Outro importante aspecto refere-se aos investimentos em educação (Lieducef), os quais se comportam como um imposto e, portanto, exercem influência negativa sobre a taxa de crescimento da produtividade de longo prazo, ou seja, agem no curto prazo como um custo social, conforme colunas (1)-(5) da Tabela 7. Este impacto pode ser medido pelo coeficiente da variável Lieducef, que indica que para cada $1 \%$ de aumento nos gastos em educação proporcional ao PIB, a taxa de crescimento média da produtividade dos Estados brasileiros reduz entre 1,3\% e 3,9\%, o que equivale a uma taxa oscilando entre $-1,2 \%$ e $1,4 \%$, implicando, portanto um impacto considerável na produtividade. $^{10}$

10 Este resultado é interessante, pois quando extrapolado em nível de países pode, por exemplo, ser um indicativo do motivo pelo qual os países em desenvolvimento apresentaram queda em suas taxas de crescimento na década de 80 e 90 , período em que mais fizeram reformas e investiram em educação, de acordo com Easterly (2001). Assim, os investimentos em educação parecem ser uma boa explicação para a queda nas suas taxas de curto prazo de crescimento econômico, pois alocaram uma grande parte dos recursos da economia do setor produtor de bens e serviços para o setor de acumulação de capital humano. 
TABELA 7 - EDUCAÇÃO E CRESCIMENTO ECONÔMICO - EFEITOS FIXOS - VARIÁVEL DEPENDENTE: TAXA DE CRESCIMENTO DA PRODUTIVIDADE DOS ESTADOS (TCP)

\begin{tabular}{|c|c|c|c|c|c|}
\hline Variáveis & (1) & (2) & (3) & (4) & (5) \\
\hline Reducef & $\begin{array}{c}0,215 \\
(0,03)^{*}\end{array}$ & $\begin{array}{c}0,163 \\
(0,027)^{\star}\end{array}$ & $\begin{array}{c}0,206 \\
(0,025)^{*}\end{array}$ & $\begin{array}{c}0,185 \\
(0,023)^{*}\end{array}$ & $\begin{array}{c}0,185 \\
(0,025)^{*}\end{array}$ \\
\hline Reduc2ef & $\begin{array}{c}0,153 \\
(0,188)\end{array}$ & $\begin{array}{c}0,939 \\
(0,164)^{*}\end{array}$ & $\begin{array}{c}0,205 \\
(0,137)\end{array}$ & $\begin{array}{c}0,328 \\
(0,126)^{*}\end{array}$ & $\begin{array}{c}0,545 \\
(0,140)^{*}\end{array}$ \\
\hline Lieducef & $\begin{array}{l}-0,013 \\
(0,07)^{\star \star \star}\end{array}$ & $\begin{array}{l}-0,034 \\
(0,002)^{*}\end{array}$ & $\begin{array}{l}-0,038 \\
(0,003)^{*}\end{array}$ & $\begin{array}{l}-0,039 \\
(0,001)^{*}\end{array}$ & $\begin{array}{l}-0,037 \\
(0,001)^{*}\end{array}$ \\
\hline Tecimef & $\begin{array}{l}0,006 \\
(0,004)^{\star \star *}\end{array}$ & $\begin{array}{c}0,015 \\
(0,003)^{*}\end{array}$ & $\begin{array}{c}0,008 \\
(0,003)^{*}\end{array}$ & $\begin{array}{c}0,011 \\
(0,003)^{*}\end{array}$ & $\begin{array}{c}0,014 \\
(0,003)^{*}\end{array}$ \\
\hline Pcpropriaef & $\begin{array}{c}0,466 \\
(0,066)^{*}\end{array}$ & $\begin{array}{c}0,293 \\
(0,068)^{*}\end{array}$ & $\begin{array}{c}0,372 \\
(0,077)^{*}\end{array}$ & $\begin{array}{c}0,299 \\
(0,071)^{*}\end{array}$ & $\begin{array}{c}0,281 \\
(0,077)^{*}\end{array}$ \\
\hline Tdesef & $\begin{array}{c}1,00 \\
(0,11)^{*}\end{array}$ & $\begin{array}{c}0,42 \\
(0,13)^{\star}\end{array}$ & $\begin{array}{c}0,82 \\
(0,17)^{\star}\end{array}$ & $\begin{array}{c}0,69 \\
(0,13)^{*}\end{array}$ & $\begin{array}{c}0,49 \\
(0,13)^{*}\end{array}$ \\
\hline$Y_{k}$ ef & $\begin{array}{l}-0,000011 \\
(1,1 \mathrm{e}-06)^{*}\end{array}$ & $\begin{array}{l}-7,7 e-06 \\
(2,8 \mathrm{e}-06)^{*}\end{array}$ & $\begin{array}{l}-0,00001 \\
(1,7 e-06)^{*}\end{array}$ & $\begin{array}{l}-8,8 \mathrm{e}-06 \\
(1,9 \mathrm{e}-06)\end{array}$ & $\begin{array}{l}-5,8 \mathrm{e}-06 \\
(2,6 \mathrm{e}-06)^{* *}\end{array}$ \\
\hline Ano*Sul & $\begin{array}{c}0,000016 \\
(5,5 \mathrm{e}-06)^{*}\end{array}$ & $\begin{array}{c}0,000013 \\
(5,6 \mathrm{e}-06)^{*}\end{array}$ & $\begin{array}{r}0,000014 \\
(5,7 \mathrm{e}-06)^{*}\end{array}$ & $\begin{array}{c}0,000013 \\
(5,5 \mathrm{e}-06)^{*}\end{array}$ & $\begin{array}{r}0,000015 \\
(5,4 \mathrm{e}-06)^{*}\end{array}$ \\
\hline Ano*Centro-Oeste & $\begin{array}{c}0,000011 \\
(4,3 e-06)^{*}\end{array}$ & $\begin{array}{r}0,000018 \\
(6,8 \mathrm{e}-06)^{*}\end{array}$ & $\begin{array}{r}0,000017 \\
(4,7 e-06)^{*}\end{array}$ & $\begin{array}{r}0,000020 \\
(4,2 \mathrm{e}-06)^{*}\end{array}$ & $\begin{array}{r}0,000026 \\
(6,1 \mathrm{e}-06)^{*}\end{array}$ \\
\hline Ano*Nordeste & $\begin{array}{l}-0,00013 \\
(4,8 \mathrm{e}-06)^{*}\end{array}$ & $\begin{array}{r}0,000018 \\
(5,7 e-06)^{*}\end{array}$ & $\begin{array}{c}8,2 \mathrm{e}-06 \\
(4,9 \mathrm{e}-06)^{\star \star *}\end{array}$ & $\begin{array}{l}-0,000016 \\
(4,7 e-06)^{*}\end{array}$ & $\begin{array}{r}0,000021 \\
(5,6 \mathrm{e}-06)^{*}\end{array}$ \\
\hline Ano*Norte & $\begin{array}{l}-0,000033 \\
(4,9 \mathrm{e}-06)^{*}\end{array}$ & $\begin{array}{l}-5,1 e-06 \\
(5,8 e-06)\end{array}$ & $\begin{array}{l}-0,000011 \\
(5,9 \mathrm{e}-06)^{\star \star \star}\end{array}$ & $\begin{array}{l}-1,5 e-06 \\
(5,8 \mathrm{e}-06)\end{array}$ & $\begin{array}{c}2,2 \mathrm{e}-06 \\
(6,4 \mathrm{e}-06)\end{array}$ \\
\hline Constante & $\begin{array}{l}-3,45 \\
(3,73)\end{array}$ & $\begin{array}{l}-5,56 \\
(3,96)\end{array}$ & $\begin{array}{l}-5,17 \\
(3,87)\end{array}$ & $\begin{array}{l}-6,34 \\
(3,46)^{\star * *}\end{array}$ & $\begin{array}{l}-5,41 \\
(3,96)\end{array}$ \\
\hline Pobreef & & $\begin{array}{c}2,336 \\
(0,301)^{*}\end{array}$ & & & \\
\hline Médiaef & & & $\begin{array}{c}0,592 \\
(0,092)^{*}\end{array}$ & & \\
\hline Ricoef & & & & $\begin{array}{l}-0,589 \\
(0,067)^{*}\end{array}$ & \\
\hline Ginief & & & & & $\begin{array}{l}-0,800 \\
(0,101)^{*}\end{array}$ \\
\hline $\mathrm{N}$ & 104 & 104 & 104 & 104 & 104 \\
\hline $\mathrm{R}^{2}$ & - & - & - & - & - \\
\hline
\end{tabular}

Obs: ${ }^{*}$ significante a $1 \% ;{ }^{*}$ significante a $5 \%$ e *** significante a $10 \% ; \mathrm{N}$ é o número de observações.

Modelo estimado considerando autocorrelação específica de painéis de dados, heterocedasticidade e dependência seccional.

Fonte: Calculada pelos autores. 
Devido a esse impacto negativo, decidimos verificar se o comportamento desse imposto possui o efeito preconizado por Barro (1990), no qual os impostos investidos em infra-estrutura possuem uma relação não-linear de $U$ invertido com a taxa de crescimento da economia. Como aqui estamos investigando a taxa de crescimento da produtividade, queremos verificar se um aumento nos investimentos implica que, a partir de algum nível, as economias dos Estados obtêm efeitos positivos e diretos sobre a taxa de crescimento de suas respectivas produtividades. Para controlar os efeitos de diferenças remanescentes entre os Estados, utilizamos as variáveis de desigualdade de renda, conforme proposto na equação (22). Portanto, do ponto de vista econométrico, estas variáveis têm como função controlar os efeitos fixos adicionais dos Estados brasileiros.

Nas colunas (2)-(5) da Tabela 8, observa-se que a variável Lieduc2 se apresenta como significante e com o sinal negativo, ou seja, os aumentos em impostos para aplicação em infra-estrutura educacional geram efeitos crescentes de impacto negativo na taxa de crescimento de longo prazo da economia. Isto significa que o efeito de U invertido não se verifica nos Estados brasileiros, com relação aos impostos destinados à educação.

É importante frisar que os benefícios resultantes desse investimento, ou seja, a melhoria do nível educacional de empregados e empregadores, são amplamente favoráveis à economia no longo prazo, conforme atestam os coeficientes da variável Reduc, que em todas as especificações estimadas aqui se apresentam como positivas e significantes. Em síntese, os investimentos em educação apresentam-se com impacto negativo, mas o efeito positivo de melhoria do nível de escolaridade dos empregados em relação ao dos empregadores resulta em aumentos da taxa de crescimento da produtividade.

Em suma, maior acesso à educação pelos empregados vis-à-vis empregadores, à tecnologia e às políticas que melhoram sua participação, especialmente os $40 \%$ mais pobres, na renda agregada, tende a auxiliar o crescimento da produtividade dos Estados brasileiros. Além disso, os impostos para financiar investimentos em educação são o custo social que deve ser pago para se obter maior crescimento da produtividade advinda do capital humano no longo prazo; portanto, a atenção quanto a sua alocação e eficiência são as melhores formas de redução deste custo social. 
TABELA 8 - INVESTIMENTO EM EDUCAÇÃO E CRESCIMENTO ECONOAMICO - EFEITOS FIXOS - VARIÁVEL DEPENDENTE: TAXA DE CRESCIMENTO DA PRODUTIVIDADE DOS ESTADOS (TCP)

\begin{tabular}{|c|c|c|c|c|c|}
\hline Variáveis & $(1)$ & $(2)$ & (3) & (4) & (5) \\
\hline Reducef & $\begin{array}{c}0,235 \\
(0,020)^{*}\end{array}$ & $\begin{array}{c}0,147 \\
(0,016)^{*}\end{array}$ & $\begin{array}{c}0,244 \\
(0,016)^{*}\end{array}$ & $\begin{array}{c}0,139 \\
(0,003)^{*}\end{array}$ & $\begin{array}{c}0,193 \\
(0,024)^{*}\end{array}$ \\
\hline Lieducef & $\begin{array}{l}-0,008 \\
(0,015)\end{array}$ & $\begin{array}{l}-0,176 \\
(0,031)^{*}\end{array}$ & $\begin{array}{l}-0,191 \\
(0,021)^{*}\end{array}$ & $\begin{array}{l}-0,321 \\
(0,031)^{*}\end{array}$ & $\begin{array}{l}-0,193 \\
(0,023)^{*}\end{array}$ \\
\hline Lieducef2 & $\begin{array}{l}-0,0015 \\
(0,002)\end{array}$ & $\begin{array}{l}-0,0026 \\
(0,005)^{*}\end{array}$ & $\begin{array}{l}-0,030 \\
(0,003)^{*}\end{array}$ & $\begin{array}{l}-0,054 \\
(0,006)^{*}\end{array}$ & $\begin{array}{l}-0,029 \\
(0,004)^{*}\end{array}$ \\
\hline Tecimef & $\begin{array}{c}0,007 \\
(0,003)^{* *}\end{array}$ & $\begin{array}{c}0,002 \\
(0,003)\end{array}$ & $\begin{array}{c}0,003 \\
(0,004)\end{array}$ & $\begin{array}{c}0,005 \\
(0,005)\end{array}$ & $\begin{array}{c}0,004 \\
(0,003)\end{array}$ \\
\hline Pcpropriaef & $\begin{array}{c}0,448 \\
(0,065)^{*}\end{array}$ & $\begin{array}{c}0,079 \\
(0,098)^{*}\end{array}$ & $\begin{array}{c}0,598 \\
(0,027)^{*}\end{array}$ & $\begin{array}{c}0,407 \\
(0,090)^{*}\end{array}$ & $\begin{array}{c}0,350 \\
(0,057)^{*}\end{array}$ \\
\hline Tdesef & $\begin{array}{c}1,07 \\
(0,09)^{*}\end{array}$ & $\begin{array}{l}0,49 \\
(0,21)^{\star *}\end{array}$ & $\begin{array}{c}1,14 \\
(0,10)^{*}\end{array}$ & $\begin{array}{c}1,12 \\
(0,18)^{*}\end{array}$ & $\begin{array}{c}0,84 \\
(0,11)^{\star}\end{array}$ \\
\hline$Y_{k} e f$ & $\begin{array}{l}-0,000011 \\
(9,1 e-07)^{*}\end{array}$ & $\begin{array}{l}-0,000014 \\
(1,6 \mathrm{e}-06)^{*}\end{array}$ & $\begin{array}{l}-9,4 \mathrm{e}-06 \\
(5,6 \mathrm{e}-07)^{*}\end{array}$ & $\begin{array}{l}-0,000010 \\
(1,6 \mathrm{e}-06)^{\star}\end{array}$ & $\begin{array}{l}-0,000012 \\
(1,5 e-06)^{*}\end{array}$ \\
\hline Ano*Sul & $\begin{array}{r}0,000018 \\
(5,8 \mathrm{e}-06)^{*}\end{array}$ & $\begin{array}{c}5,5 e-06 \\
(4,4 e-06)\end{array}$ & $\begin{array}{r}0,000011 \\
(3,4 \mathrm{e}-06)^{*}\end{array}$ & $\begin{array}{c}7,2 \mathrm{e}-06 \\
(3,3 \mathrm{e}-06)^{\star *}\end{array}$ & $\begin{array}{r}0,000011 \\
(3,7 e-06)^{*}\end{array}$ \\
\hline Ano*Centro-Oeste & $\begin{array}{r}0,000012 \\
(4,3 e-06)^{*}\end{array}$ & $\begin{array}{l}-2,7 e-06 \\
(4,3 e-06)\end{array}$ & $\begin{array}{c}6,7 e-06 \\
(4,0 e-06)\end{array}$ & $\begin{array}{l}-7,1 e-06 \\
(3,4 e-06)\end{array}$ & $\begin{array}{l}-2, e-06 \\
(4,8 e-06)\end{array}$ \\
\hline Ano*Nordeste & $\begin{array}{l}-9,6 \mathrm{e}-06 \\
(4,6 \mathrm{e}-06)^{\star *}\end{array}$ & $\begin{array}{l}-8,5 \mathrm{e}-06 \\
(4,4 \mathrm{e}-06)^{\star *}\end{array}$ & $\begin{array}{l}-0,000020 \\
(4,9 e-06)^{*}\end{array}$ & $\begin{array}{l}-0,000013 \\
(4,1 \mathrm{e}-06)^{*}\end{array}$ & $\begin{array}{l}-0,000011 \\
(4,9 \mathrm{e}-06)^{\star *}\end{array}$ \\
\hline Ano*Norte & $\begin{array}{l}-0,000032 \\
(4,5 e-06)^{*}\end{array}$ & $\begin{array}{l}-0,000023 \\
(6,2 \mathrm{e}-06)^{*}\end{array}$ & $\begin{array}{l}-0,000047 \\
(4,7 e-06)^{*}\end{array}$ & $\begin{array}{l}-0,000040 \\
(6,3 e-06)^{*}\end{array}$ & $\begin{array}{l}-0,000034 \\
(4,8 \mathrm{e}-06)^{*}\end{array}$ \\
\hline Constante & $\begin{array}{c}0,0022 \\
(0,031)\end{array}$ & $\begin{array}{c}0,296 \\
(0,057)^{*}\end{array}$ & $\begin{array}{c}0,346 \\
(0,039)^{*}\end{array}$ & $\begin{array}{c}0,570 \\
(0,062)^{*}\end{array}$ & $\begin{array}{c}0,325 \\
(0,051)^{*}\end{array}$ \\
\hline Pobrer & & $\begin{array}{c}1,311 \\
(0,496)^{*}\end{array}$ & & & \\
\hline Médiar & & & $\begin{array}{c}0,174 \\
(0,183)\end{array}$ & & \\
\hline Ricor & & & & $\begin{array}{l}-4,378 \\
(0,715)^{*}\end{array}$ & \\
\hline Ginir & & & & & $\begin{array}{l}-0,634 \\
(0,173)^{*}\end{array}$ \\
\hline $\mathrm{N}$ & 104 & 78 & 78 & 78 & 78 \\
\hline $\mathrm{R}^{2}$ & - & & & & \\
\hline
\end{tabular}

Fonte: Calculado pelos autores. Obs: ${ }^{*}$ significante a $1 \% ;{ }^{*}$ significante a $5 \% \mathrm{e}^{* *}$ significante a $10 \%$; $\mathrm{N}$ é o número de observações. 


\section{CONCLUSÃO}

Neste artigo propomos um modelo teórico que considera os efeitos da distribuição de renda, dos níveis de educação dos empregados e empregadores, dos investimentos em educação e do nível tecnológico sobre a taxa de crescimento da produtividade da economia. Os efeitos negativos da distribuição de renda foram confirmados nos testes econométricos, em especial quando consideramos o índice de Gini e a variável de concentração de renda dos $10 \%$ mais ricos. No entanto, verificamos que a proporção de renda dos $40 \%$ mais pobres e a da classe média ampla indicam os benefícios das políticas de redistribuição de renda em favor destas classes sobre a taxa de crescimento da produtividade. Quanto à forma de implementação, segundo o modelo teórico, para que ocorra uma associação positiva dessas classes com o crescimento da produtividade, deve permitir às mesmas um acesso maior à educação e à tecnologia.

Os resultados econométricos demonstraram que a acumulação de capital humano pelos empregados vis-à- $v i s$ empregadores causa um efeito positivo e benéfico sobre a taxa de crescimento da produtividade dos Estados brasileiros. Este efeito pode ser maior com o acesso à educação por parte dos trabalhadores que estão na faixa de renda dos $40 \%$ mais pobres, indicando a importância de políticas específicas para o crescimento e o desenvolvimento econômico de longo prazo da economia dos Estados brasileiros.

No entanto, os efeitos negativos de investimentos de curto prazo em educação sobre a taxa de crescimento da produtividade parecem comprovar a tese de que o investimento em educação causa realocação imediata de recursos do setor produtor de bens e serviços para o setor de acumulação de capital humano. Este efeito pode ser um dos fatores inibidores de maiores investimentos em educação nos Estados brasileiros. A inspeção de aumentos maiores de investimentos em educação demonstra que estes continuam a ser negativos, ou seja, maiores investimentos causam maior realocação, explicando o fato de estes investimentos não ocorrerem com a mesma intensidade nos Estados.

Além disso, o grau de desenvolvimento tecnológico da economia foi capturado através de duas variáveis: tecnologia importada e nível de estoque de capital físico, em que ambas se apresentam como proxies para o nível de desenvolvimento dos Estados brasileiros. Estas variáveis demonstram que o grau de interação com tecnologias do exterior é importante para o crescimento econômico, mas ao mesmo tempo maior nível de capital reduz a taxa de crescimento no longo prazo. Estes resultados podem ser indicativos de convergência entre os Estados. No entanto, estes resultados somente são válidos se considerarmos os demais efeitos das variáveis como constantes. Os Estados que compõem as regiões Sul e Centro-Oeste apresentaram uma tendência de crescimento maior em relação à região Sudeste, mesmo depois de termos controlado 
os efeitos fixos que os diferenciam. Assim, é possível que nestes Estados venham ocorrendo mudanças estruturais ao longo do tempo, que de alguma forma estão lhes beneficiando relativamente aos demais. Infelizmente, a região Nordeste se apresenta com uma tendência de crescimento de longo prazo inferior ao da região Sudeste, o que pode indicar que, no período em questão, esta região não caminhou na mesma direção das demais. Portanto, a convergência de longo prazo prevista anteriormente devido ao nível tecnológico pode não ter nenhum efeito em função dos demais fatores.

Em geral, as estimativas econométricas suportaram as características do modelo teórico para a economia brasileira, em que os níveis educacionais dos empregados e empregadores apresentam-se como importantes para a taxa de crescimento da produtividade dos Estados. No entanto, a desigualdade de renda tem o efeito de gerar maior nível de impostos; ainda que estes impostos sejam totalmente investidos em educação, haverá implicações negativas para o crescimento da economia no curto prazo. Portanto, a consciência deste fato é um importante aspecto para que a realização dos investimentos em educação seja contínua e duradoura para se obter o capital humano esperado e, no futuro, estes irão gerar crescimento da produtividade de longo prazo, compensando este custo social de curto prazo.

\section{REFERENNCIAS BIBLIOGRÁFICAS}

Acemoglu, D.; Johnson, S.; Robinson, J. A.; Yared, P. From Education to Democracy? The American Economic Review: Papers and Proceedings, v. 95, n. 2, p.44-49, 2005 .

Alesina, A.; Perotti, R. A Political Economy of Growth: A Critical Survey of Recent Literature. World Bank Economic Review, v. 8, n. 3, p. 350-371, 1994.

Alesina, A.; Rodrik, D. Distributive Politics and Economic Growth. Quarterly Journal of Economics, v. 109, n. 2, p. 465-490,1994.

Almeida, L. O.; Guilhoto, J. J. M. Crescimento Econômico e Distribuição de Renda: uma análise a partir das estruturas econômicas do Brasil contemporâneo. In: Anais XXXIV Encontro Nacional de Economia. Salvador, 2006.

Arellano, M. Panel Data Econometrics: Advanced Texts in Econometrics. Oxford: Oxford Univeristy Press, 2003.

Azzoni, C. R. Economic Growth and Regional Income Inequality in Brazil. The Annals of Regional Science, v.35, n.1, p.133-152, 2001.

Azzoni, C. R.; Servo, L.M.S. Education, Cost of Living and Regional Wage Inequality in Brazil. Papers in Regional Science, v.81, n.1, p.157-175, 2002.

Bacha, E.; Taylor, L. Brazilian Income Distribution in the 1960s: Facts, Model Results and the Controversy. Journal of Development Studies, v.14, p. 271-297, 1978. 
Baltagi, B. H.; Li, Q. A Joint Test for Serial Correlation and Random Effects. Statistics and Probability Letters, v. 11, n. 1, p. 277-280, 1991.

Testing $\mathrm{AR}(\mathrm{l})$ Against MA(1) Disturbances in an Error Component Model. Journal of Econometrics, v.68, n.1, p.133-151, 1995.

Banerjee, A. V.; Duflo, E. Inequality and Growth: What Can the Data Say? Journal of Economic Growth, v.8, n.3, p. 267-299, 2003.

Barro, R. J. Government Spending in a Simple Model of Endogenous Growth. Journal of Political Economy, v.98, n.5, p.103-125, 1990.

Determinants of Economic Growth: A Cross Country Empirical Study. Cambridge: MIT Press, 1998.

Barros, R. P.; Mendonça, R. A Evolução do Bem-Estar e da Desigualdade no Brasil Desde 1960. Rio de Janeiro: IPEA, 1992 (Texto para Discussão 28 -Série IPEA.)

Os Determinantes da Desigualdade no Brasil. In: IPEA, A Economia Brasileira em Perspectivas. Rio de Janeiro: IPEA, 1996.

Bonelli, R.; Sedlacek, G. L. Distribuição de Renda: Evolução no Último Quarto de Século. In: Sedlacek, G. L.; Barros, R. P, Mercado de Trabalbo e Distribuição e Renda: uma coletânea. Rio de Janeiro: IPEA,1989.

Bourguignon, F. V. The Distributional Effects of Growth: Case Studies vs Cross-Country Regressions. Working Paper 2002-23. DELTA - Département et Laboratoire D'Economie Théorique et Appliqueé, Paris, 2002.

Breusch, T. S.; Pagan, A. R. The LM Test and Its Application to Model Specification in Econometrics. Review of Economic Studies, v.47, n.1, p. 239-254, 1980.

Calmon, P. P.; Conceição, P.; Galbraith, J. K. Inequality and Industrial Wage Change in Brazil. The University of Texas Inequality Project, 1999. (UTIP Working Paper 12)

.; Cantú, V. G.; Hibert, A. The Evolution of Industrial Earnings Inequality in Mexico and Brazil. Review of Development Economics, v.4, n.2, p.194-203, 2000.

Cardoso, E.; Barros, R. M.; Urani, A. Inflation and Unemployment as Determinant of Inequality in Brazil in the 80's. In: Dornbusch, R.; Edwards, S. (eds). Reform, Recovery and Growth: Latin America and the Middle-East. Chicago: University Chicago Press, 1995.

Castello, A.; Domenech, R. Human Capital Inequality and Economic Growth: Some New Evidence. Economic Journal, 112, C187- C200, 2002.

Chiang, A. C. Dynamic Optimization. New York: McGraw-Hill, 1992.

Deininger, K.; Squire, L. A New Data Set Measuring Income Inequality. World Bank Economic Review, v.10, n. 3, p. 565-591, 1996.

. New Ways of Looking at Old Issues: Inequality and Growth. Journal of Development Economics, v.57, n.1, p. 259-287, 1998. 
Dias, J. An Economic Growth Model Based on Entrepreneurial Knowledge. Revista Econômica do Nordeste, v.32, Número Especial, p.740-759, 2001.

Educational System, Income Inequality and Growth: The Median Voter's Decision. Estudos Econômicos, v.35, n.1, p.81-100, 2005a.

; Dias, M. H. A. Educação dos Jovens, Analfabetismo e o Custo Governo: aplicaçóes econométricas para o Brasil. Anais do XXII Encontro Nacional de Economia-ANPEC. João Pessoa, 2004.

; McDermott, J. Education, Institutions, and Growth: The Role of Entrepreneurs. Journal of Development Economics, v. 80, n.1, p. 299-328, 2006.

Easterly, W. The Lost Decades: Developing Countries' Stagnation in Spite of Policy Reform 1980-1998. Journal of Economic Growth, v.6, n.1, p.135-157, 2001.

. The Middle Class Consensus and Economic Development. Journal of Economic Growth, v.6, n.2, p. 317-335, 2001 a.

; Rebelo, S. Fiscal Policy and Economic Growth: An Empirical Investigation. Journal of Monetary Economics, v.32,n. 2, p. 417-458, 1993.

Eicher, T.; Garcia-Peñaloza, C. Inequality and Growth: The Dual Role of Human Capital in Development. Working Paper University of Washington. Washington, DC, 1999.

Elbers, C., Lanjouw, J. O. ; Lanjouw, P. Poverty and Inequality in Brazil: New Estimates from Combined PPV-PNAD Data. World Bank PREM Inequality Thematic Group - Discussion Paper. Washington, DC, 2004.

Ferreira, F. H. G. Os Determinantes da Desigualdade de Renda no Brasil: luta de classes ou heterogeneidade educacional? Rio de Janeiro: PUC, 1999 (Texto para Discussão)

; Lanjouw, P. E.; Neri, M. A Robust Poverty Profile for Brazil Using Multiple Data Sources. Revista Brasileira de Economia, v.57, n. 2, p. 59-92, 2003.

; Litchfield, J. A. Education or inflation?: The Micro and Macroeconomics of the Brazilian Income Distribution During 1981-1995. Cuadernos de Economia, 38, p. 209-238, 2001.

Ferreira, P. C.; Ellery Jr., G. Convergência entre a Renda Per Capita dos Estados Brasileiros. Revista de Econometria, v.16, n.1, p.83-103, 1996.

Forbes, K. A Reassessement of the Relationship between Inequality and Growth. The American Economic Review, v.90, n. 4, p. 869-887, 2000.

Glomm, G.; Ravikumar, B. Public Versus Private Investment in Human Capital: endogenous growth and income inequality. Journal of Political Economy, v.100, n. 4, p. 818-834, 1992.

Greene, W. H. Econometric Analysis. Upper Sadle River, NJ: Prentice-Hall, 2000. 
Hadri, K. Testing for Stationarity in Heterogeneous Panel Data. Econometrics Journal, v.3, n. 1, p.148-161, 2000.

Hausman, J. A. Specification Tests in Econometrics. Econometrica, v.46, n.5, p.12511271, 1978.

Hoffman, R. Evolução da Distribuição da Renda no Brasil, entre Pessoas e entre Famílias, 1979/86. In: Sedlacek, G. L.; Barros R. P, Mercado de Trabalho e Distribuição de Renda: uma coletânea. Rio de Janeiro: IPEA, 1989.

. As Transferências não são a Principal Causa da Redução da Desigualdade. Econômica ,v.7, n. 2, p.335-341, 2005.

Hsiao, C. Analysis of Panel Data. Cambridge: Cambridge University Press, 2003.

IPEA. Economia em Perspectiva 1998. Rio de Janeiro: IPEA, 1998.

Jones, C. I. Time Series Tests of Endogenous Growth Models. Quarterly Journal of Economics, v.110, n. 3, p.495-525, 1995.

Judge, G, G.; Griffiths, W. E.; Hill, R. C.; Lutkepohl, H.; Lee, T.C. The Theory and Practice of Econometrics. New York: John Wiley and Sons, 1985.

Kakwani, N.; Neri, M.; Son, H. H. Pro-Poor Growth and Social Programmes in Brazil. Fifth General Meeting of the Poverty and Economic Policy Research Network. Addis Ababa, 2006.

Kiefer, N. M. Estimation of Fixed Effects Models for Time Series of Cross-Section with Arbitrary Intertemporal Covariance. Journal of Econometrics, v.14, n.1, p.195-202, 1980.

Kuznets, S. Economic Growth and Income Inequality. The American Economic Review, v. 45 , n.1, p.1-28, 1955.

Lam, D.; Levison, D. Declining Inequality in Schooling in Brazil and Its Effects on Inequality in Earnings. Journal of Development Economics, v.37, n.1, p.199-225, 1991.

Langoni, C. Distribuição de Renda e Crescimento no Brasil. Rio de Janeiro:Editora Expressão e Cultura, 1973.

Leal, C. S. ; Werlang, S. Educação e Distribuição de Renda. In: Camargo, José Marcio; Giambiagi, Fabio (Eds), Distribuição de Renda no Brasil. Rio de Janeiro: Paz e Terra, 1991.

Lucas Jr., R. E. On the Mechanics of Development Planning. Journal of Monetary Economics, v.22, n. 1, p.3-42, 1988.

Milanovic, B. The Median-Voter Hyphotesis, Income Inequality, and Income Redistribution: An Empirical Test with the Required Data. European Journal of Political Economy, v. 16, n. 1, p. 367-410, 2000.

Mossi, M. B.; Aroca, P.; Fernandez, I. J.; Azzoni, C. R. Growth Dynamics and Space in Brazil. International Regional Science Review, v. 26, n.3, p.393-418, 2003. 
Panizza, U. Income Inequality and Economic Growth: Evidence from American Data. Journal of Economic Growth, v.7, n.1, p. 25-41, 2002.

Partridge, M. D. Is Inequality Harmfull for Growth? Comment. The American Economic Review, v.87, n.5, p.1019-1032, 1997.

. Does Income Distribution Affect U.S. State Economic Growth? Journal of Regional Science, v.45, n.2, p.363-394, 2005.

Peasaran, M. H. A simple Panel Unit Root Test in the Presence of Cross Section Dependence. University of Southern California Working Paper, 2003.

Perotti, R. Growth, Income Distribution, and Democracy: What the Data Say. Journal of Economic Growth, v.1, n.1, p.149-188, 1996.

Person, T.; Tabellini, G Is Inequality Harmfull for Growth? The American Economic Review, v.84, n.3, p.600-621, 1994.

Ramos, L. A Distribuição de Rendimentos no Brasil 1976/85. Rio de Janeiro: IPEA, 1993.(Texto para Discussão 141 - Série IPEA.)

. Interindustry Wage Differentials. Rio de Janeiro: IPEA, 1995. (Texto para Discussão 374- Série IPEA)

Romer, D. Advanced Macroeconomics. $2^{\text {nd }}$ Edition. New York: McGraw-Hill, 2006.

Sen, A. Editorial: Human Capital and Human Capability. World Development, v. 25, n.12, p.1959-1961, 1997.

Souza, A. M. Educational Policies: Efficiency and Equity Issues. Revista Brasileira de Economia, v.48, n.4, p.411-420, 1994.

Sylvester, K. Income Inequality, Education Expenditures, and Growth. Journal of Development Economics, v.63, n.2, p.379-398, 2000.

. Can Education Expenditure Reduce Income Inequality? Economics of Education Review, v.21, n.1, p. 43-52, 2002.

Temple, J. The New Growth Evidence. Journal of Economic Literature, v.37, n.1, p.112-156, 1999.

Walde, K. Egalitarian and Elitist Education Systems as the Basis for International Differences in Wage Inequality. European Journal of Political Economy, v.16, n.3, p. $445-468,2000$.

Wolf, E. N. Human Capital Investiment and Economic Growth: Exploring CrossCountry Evidence. Structural Change and Economic Dynamics, v.11, n.4, p.433$472,2000$.

Wooldridge, J. M. Econometric Analysis of Cross Section and Panel Data. Cambridge: MIT Press, 2002.

Zini Jr., A. A. Regional Income Convergence in Brazil and Its Socio-Economic Determinants. Economia Aplicada, v.2, n.2, p.383-411, 1998. 


\section{APENDICE}

TABELA AI - DESCRIÇÃO ESTATÍSTICA DOS DADOS DOS ESTADOS BRASILEIROS: 1992-1996

\begin{tabular}{lcccccc}
\hline Variável & $\begin{array}{c}\text { Média } \\
\text { Geral }\end{array}$ & $\begin{array}{c}\text { Desvio Padrão } \\
\text { Geral }\end{array}$ & $\begin{array}{c}\text { Desvio Padrão } \\
\text { entre os } \\
\text { Estados: Painéis }\end{array}$ & $\begin{array}{c}\text { Desvio Padrão } \\
\text { dos Estados: } \\
\text { Tempo }\end{array}$ & $\begin{array}{c}\text { Valor Mínimo: } \\
\text { entre os } \\
\text { Estados }\end{array}$ & $\begin{array}{c}\text { Valor Máximo: } \\
\text { entre os } \\
\text { Estados }\end{array}$ \\
\hline Tcp & 0,027 & 0,069 & 0,029 & 0,062 & $-0,057$ & 0,084 \\
Heduc & 5,783 & 1,182 & 1,150 & 0,339 & 3,874 & 8,256 \\
Keduc & 7,814 & 1,414 & 1,288 & 0,625 & 4,294 & 9,950 \\
Reduc & 0,74 & 0,11 & 0,09 & 0,07 & 0,600 & 0,934 \\
leduc & 0,047 & 0,036 & 0,033 & 0,017 & 0,022 & 0,154 \\
Tecim & 0,001 & 0,004 & 0,003 & 0,003 & 0 & 0,015 \\
Pcpropria & 0,211 & 0,052 & 0,051 & 0,012 & 0,111 & 0,397 \\
Tdes & 0,069 & 0,024 & 0,021 & 0,011 & 0,028 & 0,119 \\
Pobre & 0,091 & 0,012 & 0,009 & 0,007 & 0,077 & 0,110 \\
Média & 0,443 & 0,034 & 0,026 & 0,023 & 0,392 & 0,516 \\
Rico & 0,465 & 0,043 & 0,034 & 0,028 & 0,372 & 0,526 \\
Gini & 0,560 & 0,034 & 0,027 & 0,021 & 0,489 & 0,600 \\
Tcpibreal & 0,038 & 0,063 & 0,023 & 0,058 & $-0,010$ & 0,100 \\
\hline
\end{tabular}

\section{A. Descrição das Variáveis:}

Tcp - Taxa de crescimento da produtividade dos trabalhadores. Divisão do PIBProduto Interno Bruto do Estado pelo Número de Trabalhadores empregados nas empresas. Portanto, não foram considerados os trabalhadores por conta própria e os desempregados. A média indica uma taxa de crescimento média no período de $2,7 \%$ ano.

Heduc - Nível de escolaridade média dos empregados em anos. Obtida através da multiplicação da freqüência pelo nível de escolaridade correspondente. A média é de 5,783 anos de escolaridade.

Keduc - Nível de escolaridade média dos empregadores. Seu cálculo foi similar à variável anterior. A sua média corresponde a 7,814 anos de escolaridade.

Reduc - A razão entre as variáveis Heduc e Keduc, sendo que sua média corresponde a que os empregados possuem em média $74 \%$ do nível de escolaridade dos empregadores. 
Ieduc - Investimento em educação como percentual do PIB dos Estados. A média dos investimentos em educação nos Estados brasileiros no período foi de $4,7 \%$ do PIB.

Tecim - Tecnologia importada como proporção do PIB de cada Estado. O valor da média corresponde a um investimento de $0,1 \%$ do PIB com a importação de tecnologias.

Pcpropria - Proporção de trabalhadores por conta própria em relação à população economicamente ativa dos Estados. O percentual de 21,1\% representa a média destes trabalhadores no período.

Tdes - Taxa de desemprego nos Estados. A média no período dos desempregados foi de $6,9 \%$.

Pobre - Participação na distribuição da renda da população que está entre o primeiro e o quarto decil, ou seja, a proporção da renda dos $40 \%$ mais pobres. A média da participação no período foi de $9,1 \%$.

Média - Participação na distribuição da renda da população que está entre o quinto e o oitavo decil, classe média ampla. A participação dos mesmos neste período foi de $44,3 \%$.

Rica - Participação na renda total dos $10 \%$ mais ricos, décimo decil. O valor 46,5\% representa média que coube a esta classe no período.

Gini - Índice de concentração de renda que mede a desigualdade de renda em geral. O valor médio do índice no período foi de 0,56 .

Tcpibreal - Taxa de crescimento do PIB real. O crescimento médio foi de $3,8 \%$ no período.

\section{B. Fonte dos Dados:}

As variáveis Tcp, Ieduc, Média, Rico, Gini e Tcpibreal têm como fonte IPEADATA (2004). As variáveis Pcpropria, Heduc e Keduc foram obtidas das PNAD-Programa Nacional de Amostra Domiciliar (1992, 1993, 1995, 1996). Os dados relativos a 1994 foram obtidos através de interpolação com os dados existentes. A fonte da variável Tecim é IPEA (1998). 